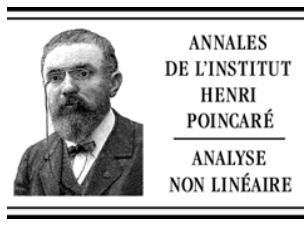

www.elsevier.com/locate/anihpc

\title{
Weak solutions to a nonlinear variational wave equation with general data
}

\author{
Ping Zhang ${ }^{\mathrm{a}, *}$, Yuxi Zheng ${ }^{\mathrm{b}}$ \\ a Academy of Mathematics and System Sciences, CAS Beijing 100080, China \\ ${ }^{\mathrm{b}}$ Department of Mathematics, Penn State University, PA 16802, USA
}

Received 24 November 2003; received in revised form 6 April 2004; accepted 22 April 2004

Available online 26 January 2005

\begin{abstract}
We establish the existence of global weak solutions to the initial value problem for a nonlinear variational wave equation $u_{t t}-c(u)\left(c(u) u_{x}\right)_{x}=0$ with general initial data $\left(u(0), u_{t}(0)\right)=\left(u_{0}, u_{1}\right) \in W^{1,2} \times L^{2}$ under the assumptions that the wave speed $c(u)$ satisfies $c^{\prime}(\cdot) \geqslant 0$ and $c^{\prime}\left(u_{0}(\cdot)\right)>0$. Moreover, we obtain high regularity for the spatial derivative $\partial_{x} u$ of the wave amplitude $u$ away from where $c^{\prime}(u)=0$. This equation arises from studies in nematic liquid crystals, long waves on a dipole chain, and a few other fields. We use Young measure method in the setting of $L^{p}$ spaces and method of renormalization to overcome the difficulty that oscillations in a sequence of approximations get amplified by the quadratic growth term of the equation. We use a high space-time estimate for $\partial_{x} u$ to handle possible concentrations. This result improves our earlier existence result for initial data in the space $W^{1, \infty} \times L^{\infty}$ to the natural space $W^{1,2} \times L^{2}$.
\end{abstract}

(C) 2004 L'Association Publications de l'Institut Henri Poincaré. Published by Elsevier B.V. All rights reserved

\section{Résumé}

Nous prouvons l'éxistence de solutions faibles globales pour le problème de Cauchy concernant une équation des ondes non variationnelles $u_{t t}-c(u)\left(c(u) u_{x}\right)_{x}=0$ avec des conditions initiales générales $\left(u(0), u_{t}(0)\right)=\left(u_{0}, u_{1}\right) \in W^{1,2} \times L^{2}$ sous l'hypothèse que la vitesse d'onde $c(u)$ vérifie $c^{\prime}(\cdot) \geqslant 0$ et $c^{\prime}\left(u_{0}(\cdot)\right)>0$. De plus, nous obtenons une régularité élevée pour la dérivée spatiale $\partial_{x} u$ de l'amplitude $u$ de l'onde loin de la zone où $c^{\prime}(u)=0$. Cette équation intervient dans l'étude des crystaux liquides nématiques, d'ondes longues dans des chaînes dipolaires et de quelques autres domaines. Nous utilisons la méthode des mesures de Young dans le contexte d'espaces $L^{p}$ et la méthode de renormalisation pour résoudre la difficulté de l'amplification par les termes à croissance quadratique de l'équation, des oscillations d'une suite d'approximations. Nous nous servons d'une estimée d'ordre élevé en espace et en temps pour $\partial_{x} u$ afin de traiter les concentrations éventuelles. Ce résultat étend nos résultats d'éxistence antérieurs pour des données initiales dans l'espace $W^{1, \infty} \times L^{\infty}$ au cas naturel de l'espace $W^{1,2} \times L^{2}$.

(C) 2004 L'Association Publications de l'Institut Henri Poincaré. Published by Elsevier B.V. All rights reserved

\footnotetext{
* Corresponding author.

E-mail addresses: zp@mail.math.ac.cn (P. Zhang), yzheng@math.psu.edu (Y. Zheng).
} 


\section{Introduction}

In this paper, we study the existence and regularity properties of weak solutions to the following nonlinear wave equation

$$
\left\{\begin{array}{l}
\partial_{t}^{2} u-c(u) \partial_{x}\left(c(u) \partial_{x} u\right)=0 \\
\left.u\right|_{t=0}=u_{0} \\
\left.\partial_{t} u\right|_{t=0}=u_{1}
\end{array}\right.
$$

where $c(\cdot)$ is a given smooth, bounded, and positive function with $c^{\prime}(\cdot) \geqslant 0$ and $c^{\prime}\left(u_{0}\right)>0, u_{0}(x) \in H^{1}(\mathbb{R})$, and $u_{1}(x) \in L^{2}(\mathbb{R})$.

One motivation for study (1.1) comes from liquid crystals. We give a brief explanation of how the equation arises in that context. For further details, see [6,8,9], and [14]. See [22] for modeling long waves on a dipole chain and [7] for another field. The mean orientation of the molecules in a nematic liquid crystal is described by a director field of unit vectors, $\mathbf{n} \in \mathcal{S}^{2}$. We consider a regime in which inertia effects dominate viscosity. The propagation of orientation waves in the director field is then modeled by a constrained variational principle

$$
\delta \iint\left\{\mathbf{n}_{t} \cdot \mathbf{n}_{t}-W(\mathbf{n}, \nabla \mathbf{n})\right\} \mathrm{d} x \mathrm{~d} t=0, \quad \mathbf{n} \cdot \mathbf{n}=1,
$$

where $W$ is the Oseen-Franck potential energy density,

$$
W(\mathbf{n}, \nabla \mathbf{n})=\alpha|\mathbf{n} \times(\mathbf{n} \times \mathbf{n})|^{2}+\beta(\nabla \cdot \mathbf{n})^{2}+\gamma(\mathbf{n} \cdot \nabla \times \mathbf{n})^{2} .
$$

This potential energy is determined (up to a null Lagrangian) by the requirement that it is invariant under reflection $\mathbf{n} \rightarrow-\mathbf{n}$ and under simultaneous rotations of the spatial variables and the director field. The positive constants $\alpha, \beta, \gamma$ are elastic constants of the liquid crystal.

A commonly used special case is the one-constant approximation in which $\alpha=\beta=\gamma$. The potential energy density then reduces to

$$
W(\mathbf{n}, \nabla \mathbf{n})=\alpha|\nabla \mathbf{n}|^{2} .
$$

The associate variational problem is identical to the variational problem for wave maps from $(1+3)$-dimensional Minkowski space into two sphere.

The simplest class of solutions for the orientational waves in a liquid crystal consists of planar deformations depending on a single space variable. The director field then has the special form

$$
\mathbf{n}=\cos u(t, x) \mathbf{e}_{x}+\sin u(t, x) \mathbf{e}_{y} .
$$

Here, the dependent variable $u$ measures the angle of the director field to the $x$-direction, $\mathbf{e}_{x}$ and $\mathbf{e}_{y}$ are coordinate vectors in the $x$ and $y$ directions, respectively. In this case, the variational principle for $\mathbf{n}$ reduces to

$$
\delta \iint\left\{u_{t}^{2}-c^{2}(u) u_{x}^{2}\right\} \mathrm{d} x \mathrm{~d} t=0,
$$

with the wave speed $c$ given by

$$
c^{2}(u)=\alpha \cos ^{2} u+\beta \sin ^{2} u,
$$


the Euler-Lagrange equation for this variational principle is (1.1). In the wave map case, we have $\alpha=\beta$, and Eq. (1.1) reduces to the standard linear wave equation.

We point out that, early in the study of (1.1), Hunter and Saxton [8] derived an asymptotic equation

$$
\partial_{t} v+u \partial_{x} v=-\frac{1}{2} v^{2}, \quad v=\partial_{x} u,
$$

for (1.1) via weakly nonlinear geometric optics. The global existence and uniqueness of solutions to the Cauchy problem is fairly complete, see [8,9] and the authors' [19]. The study of (1.3) has been very beneficial for both the blow-up result [5] and the current global existence result for the wave Eq. (1.1).

In [5], Glassey, Hunter, and Zheng have shown that singularities can form from smooth data for Eq. (1.1). When $c^{\prime}(\cdot)$ is of a single sign, some partial existence results are given in [18,20] and [21]. In [18], the authors prove the global existence of weak rarefactive solutions to (1.1) under the conditions $c^{\prime}(\cdot) \geqslant 0, R_{0} \leqslant 0, S_{0} \leqslant 0$, $\left(R_{0}, S_{0}\right) \in L^{p}(\mathbb{R}), p>3$. The notations here are that $R$ and $S$ are the Riemann invariants, see below. If the condition $c^{\prime}(\cdot) \geqslant 0$ is strengthed to $c^{\prime}(\cdot)>0$, then the condition $p>3$ can be relaxed to $p=2$. If, in addition, the initial data $u_{0} \in H^{k+1}(\mathbb{R}), u_{1} \in H^{k}(\mathbb{R})$ for some $k \geqslant 1$, then the solutions are in the same regularity class. In [20], the condition $R_{0} \leqslant 0$ is removed for the global existence of weak solutions. And in [21], we completely remove the conditions that $R_{0} \leqslant 0, S_{0} \leqslant 0$, but with $\left(R_{0}, S_{0}\right) \in L^{2}(\mathbb{R}) \cap L^{\infty}(\mathbb{R})$, in the proof of the global existence of weak solutions to (1.1).

In this paper, we establish the global existence of weak solutions for (1.1) for the wave speed $c(u)$ satisfying $c^{\prime}(\cdot) \geqslant 0$ and $c^{\prime}\left(u_{0}(\cdot)\right)>0$, and general initial data $\left(R_{0}, S_{0}\right) \in L^{2}(\mathbb{R})$. The difficulty is that the potential oscillations, in terms of DiPerna and Majda [2], get amplified unboundedly by quadratic growth terms of the equation, and the possible concentrations in the approximate solutions. We use the generalized compensated compactness ([4] or [16]), the latest development in the $L^{p}$ Young measure method of Lions [13] and Joly, Metivier and Rauch [11], the renormalization method in [1], and the techniques used in our paper [21] to treat the oscillations. We obtain high regularity for the space derivative of the wave amplitude $\partial_{x} u$ away from $c^{\prime}(u)=0$, which is the corresponding version of a Strichartz type inequality for wave equations with constant wave speed, to control the possible concentrations.

Before we present our main result, let us first give the following definition. Our notations are $\mathbb{R}^{+}=(0, \infty)$, Lip stands for Lipschitz, and

$$
R:=\partial_{t} u+c(u) \partial_{x} u, \quad S:=\partial_{t} u-c(u) \partial_{x} u, \quad \tilde{c}(\cdot):=\frac{1}{4} \ln c(\cdot),
$$

so that $\tilde{c}^{\prime}(u)=\frac{c^{\prime}(u)}{4 c(u)}$. We use $R_{0}(x)=R(0, x)$ and $S_{0}(x)=S(0, x)$.

With the above notations, we can also write (1.1) in the following form:

$$
\left\{\begin{array}{l}
\partial_{t} R-c(u) \partial_{x} R=\tilde{c}^{\prime}(u)\left(R^{2}-S^{2}\right), \\
\partial_{t} S+c(u) \partial_{x} S=\tilde{c}^{\prime}(u)\left(S^{2}-R^{2}\right), \\
\partial_{x} u=\frac{R-S}{2 c(u)}, \\
\left.R\right|_{t=0}=R_{0},\left.\quad S\right|_{t=0}=S_{0} .
\end{array}\right.
$$

Definition 1.1. We call $u(t, x)$ an admissible weak solution of (1.1) if

(1) $u(t, x) \in L^{\infty}\left(\mathbb{R}^{+}, H^{1}(\mathbb{R})\right) \cap \operatorname{Lip}\left(\mathbb{R}^{+}, L^{2}(\mathbb{R})\right)$, and

$$
\int_{\mathbb{R}}\left(\left|\partial_{t} u\right|^{2}+\left|c(u) \partial_{x} u\right|^{2}\right) \mathrm{d} x \leqslant \int_{\mathbb{R}}\left(\left|u_{1}\right|^{2}+\left|c\left(u_{0}\right) \partial_{x} u_{0}\right|^{2}\right) \mathrm{d} x ;
$$


(2) For any test function $\phi(t, x) \in C_{c}^{\infty}\left(\mathbb{R}^{+} \times \mathbb{R}\right)$, there holds

$$
\iint_{\mathbb{R}^{+} \times \mathbb{R}}\left(\partial_{t} \phi \partial_{t} u-\partial_{x} \phi c^{2}(u) \partial_{x} u-\phi c^{\prime}(u) c(u)\left(\partial_{x} u\right)^{2}\right) \mathrm{d} x \mathrm{~d} t=0 ;
$$

(3) (The entropy condition) For any $\left(t_{0}, x_{0}\right)$ with $t_{0}>0$, there always exists a positive constant $M\left(t_{0}, x_{0}\right)$ such that

$$
R(t, x) \geqslant-M\left(t_{0}, x_{0}\right), \quad S(t, x) \geqslant-M\left(t_{0}, x_{0}\right),
$$

hold in a neighborhood $\mathcal{N}\left(t_{0}, x_{0}\right)$ of $\left(t_{0}, x_{0}\right)$;

(4) $u(t, x) \rightarrow u_{0}(x)$ in $L^{2}(\mathbb{R})$ and $\partial_{t} u(t, x) \rightarrow u_{1}(x)$ in the distributional sense as $t \rightarrow 0+$.

We shall always assume that there exist two positive constant $C_{1}, C_{1}$ such that

$$
0<C_{1} \leqslant c(\cdot) \leqslant C_{2}, \quad \text { and } \quad\left|c^{(l)}(\cdot)\right| \leqslant M_{l}, \quad l \geqslant 1
$$

for some positive constants $M_{l}$.

Theorem 1.1. Let $c^{\prime}(\cdot) \geqslant 0, c^{\prime}\left(u_{0}(\cdot)\right)>0, u_{0} \in H^{1}(\mathbb{R})$, and $u_{1} \in L^{2}(\mathbb{R})$. Then (1.1) has a global admissible weak solution $u$ in the sense of Definition 1.1. Moreover,

$$
\iint_{\Omega}\left|\partial_{x} u\right|^{p} \mathrm{~d} x \mathrm{~d} t \leqslant C_{\Omega, p}, \quad \forall p<3
$$

where $\Omega$ is a small neighborhood of any point $(t, x) \in \overline{\mathbb{R}^{+}} \times \mathbb{R}$ at which $c^{\prime}(u(t, x)) \neq 0$, and $C_{\Omega, p}$ is a positive constant which depends only on $\Omega, p,\left\|u_{0}\right\|_{H^{1}}$, and $\left\|u_{1}\right\|_{L^{2}}$.

Remark 1.1. Theorem 1.1 still holds if we replace the assumptions $c^{\prime}(\cdot) \geqslant 0$ and $c^{\prime}\left(u_{0}(\cdot)\right)>0$ by $c^{\prime}(\cdot) \leqslant 0$ and $c^{\prime}\left(u_{0}(\cdot)\right)<0$. One needs only to replace the entropy condition in (1.8) by

$$
R(t, x) \leqslant M\left(t_{0}, x_{0}\right), \quad S(t, x) \leqslant M\left(t_{0}, x_{0}\right),
$$

for $(t, x) \in \mathcal{N}\left(t_{0}, x_{0}\right)$. One can check the proof of Theorem 1.1 for details.

Remark 1.2. Suppose that $c^{\prime}$ keeps sign, and $\left(R_{0}, S_{0}\right) \in L^{\infty}(\mathbb{R})$, then by [21], we know that the following ordinary differential equations have global solutions $\Phi_{t}^{ \pm}(x) \in \operatorname{Lip}([0, \infty) \times \mathbb{R})$ :

$$
\left\{\begin{array}{l}
\frac{\mathrm{d} \Phi_{t}^{ \pm}(x)}{\mathrm{d} t}= \pm c\left(u\left(t, \Phi_{t}^{ \pm}(x)\right)\right) \\
\Phi_{0}^{ \pm}(x)=x .
\end{array}\right.
$$

But here as the initial data $\left(R_{0}, S_{0}\right) \in L^{2}(\mathbb{R})$, the entropy condition (1.8) is not enough to prove this result for (1.11). Actually we do not even know that (1.11) has solutions $\Phi_{t}^{ \pm}(x) \in C([0, \infty) \times \mathbb{R})$.

Remark 1.3. Motivated by [9] and [19], we point out that we expect multiple weak solution to problem (1.1). Our weak solutions in Theorem 1.1 are solutions of the dissipative type, because the entropy condition (1.8) guarantees that: On almost all the blow-up points $(\tau, y), R(t, x) \rightarrow+\infty$ as $(t, x) \rightarrow(\tau, y)$, and similarly for $S$. But in the construction of the conservative weak solutions to (1.3) (see [9]), $v(t, x) \rightarrow-\infty$ as $t \rightarrow \tau-$ and $v(t, x) \rightarrow+\infty$ as $t \rightarrow \tau+$, if $\tau$ is the blow-up time of the solution. We plan to explore the uniqueness issue in future work.

In the following, we will try to present our proof as general as we can. Actually only one step in the proof of Theorem 1.1 uses the assumptions that $c^{\prime}(\cdot) \geqslant 0$ and $c^{\prime}\left(u_{0}(\cdot)\right)>0$. We will point the step later (see Remark 3.1). 


\section{Approximate solutions and uniform estimates}

Similar to [19-21], let us define for $\varepsilon>0$

$$
Q_{\varepsilon}(\xi):= \begin{cases}\frac{1}{\varepsilon}\left(\xi-\frac{1}{2 \varepsilon}\right), & \xi \varepsilon \geqslant 1, \\ \frac{1}{2} \xi^{2}, & \xi \varepsilon<1 .\end{cases}
$$

Let us also use the notation $\zeta^{+}:=\max (0, \zeta)$ and $\zeta^{-}:=\min (0, \zeta)$. We now define the approximate solution sequence by the equations

$$
\left\{\begin{array}{l}
\partial_{t} R^{\varepsilon}-c\left(u^{\varepsilon}\right) \partial_{x} R^{\varepsilon}=\tilde{c}^{\prime}\left(u^{\varepsilon}\right)^{+}\left(2 Q_{\varepsilon}\left(R^{\varepsilon}\right)-\left(S^{\varepsilon}\right)^{2}\right)+\tilde{c}^{\prime}\left(u^{\varepsilon}\right)^{-}\left(2 Q_{-\varepsilon}\left(R^{\varepsilon}\right)-\left(S^{\varepsilon}\right)^{2}\right), \\
\partial_{t} S^{\varepsilon}+c\left(u^{\varepsilon}\right) \partial_{x} S^{\varepsilon}=\tilde{c}^{\prime}\left(u^{\varepsilon}\right)^{+}\left(2 Q_{\varepsilon}\left(S^{\varepsilon}\right)-\left(R^{\varepsilon}\right)^{2}\right)+\tilde{c}^{\prime}\left(u^{\varepsilon}\right)^{-}\left(2 Q_{-\varepsilon}\left(S^{\varepsilon}\right)-\left(R^{\varepsilon}\right)^{2}\right), \\
\partial_{x} u^{\varepsilon}=\frac{R^{\varepsilon}-S^{\varepsilon}}{2 c\left(u^{\varepsilon}\right)} \\
\lim _{x \rightarrow-\infty} u^{\varepsilon}(t, x)=0, \\
\left.\left(R^{\varepsilon}, S^{\varepsilon}\right)\right|_{t=0}=\left(R_{0}, S_{0}\right)(x) .
\end{array}\right.
$$

That is, $R$ is chopped off in the first equation at $1 / \varepsilon$ if $c^{\prime}$ is positive, it is chopped off at $-1 / \varepsilon$ if $c^{\prime}$ is negative. No chop-off for $S$ in the first equation. Do the same for the second equation. For convenience, we sometimes omit the superscript $\varepsilon$ in the approximate solution sequence $\left\{\left(R^{\varepsilon}, S^{\varepsilon}, u^{\varepsilon}\right)\right\}_{\varepsilon>0}$.

Assume that $c(\cdot)$ satisfies $(1.9)$, but $c^{\prime}(\cdot)$ may change sign.

Lemma 2.1 (Solution of (2.2) with smooth data). Let $\left(R_{0}, S_{0}\right)(x) \in C_{c}^{\infty}(\mathbb{R})$. Then, problem (2.2) has a global smooth solution $(R, S)(t, x) \in L^{\infty}\left(\mathbb{R}^{+}, W^{1, \infty}(\mathbb{R})\right), u(t, x) \in L^{\infty}\left(\mathbb{R}^{+}, W^{2, \infty}(\mathbb{R})\right)$, which satisfies the energy inequalities

$$
\int\left(R^{2}+S^{2}\right)(t, x) \mathrm{d} x \leqslant \int\left(R_{0}^{2}+S_{0}^{2}\right)(x) \mathrm{d} x
$$

and

$$
\int_{0}^{\infty} \int_{\mathbb{R}}\left(c^{\prime}\left(u^{\varepsilon}\right)^{+} G_{\varepsilon}^{+}+c^{\prime}\left(u^{\varepsilon}\right)^{-} G_{\varepsilon}^{-}\right) \mathrm{d} x \mathrm{~d} t \leqslant \int\left(R_{0}^{2}+S_{0}^{2}\right)(x) \mathrm{d} x,
$$

where

$$
G_{\varepsilon}^{ \pm}:=R\left(R^{2}-2 Q_{ \pm \varepsilon}(R)\right)+S\left(S^{2}-2 Q_{ \pm \varepsilon}(S)\right)
$$

and $G_{\varepsilon}^{+} \geqslant 0$ and $G_{\varepsilon}^{-} \leqslant 0$. Moreover, if we introduce the plus and minus characteristics $\Phi_{t}^{ \pm}(b)$ as

$$
\left\{\begin{array}{l}
\frac{\mathrm{d}}{\mathrm{d} t} \Phi_{t}^{ \pm}= \pm c\left(u\left(t, \Phi_{t}^{ \pm}\right)\right) \\
\left.\Phi_{t}^{ \pm}\right|_{t=0}=b
\end{array}\right.
$$

then, we have the energy inequality in a characteristic cone

$$
\int_{a}^{d} R^{2}\left(t_{a}^{+}(y), y\right) \mathrm{d} y+\int_{d}^{b} S^{2}\left(t_{b}^{-}(y), y\right) \mathrm{d} y \leqslant \frac{1}{2} \int_{a}^{b}\left(R_{0}^{2}+S_{0}^{2}\right)(x) \mathrm{d} x,
$$

where $a<b$, and $d$ is where the two characteristics $\Phi_{t}^{+}(a)$ and $\Phi_{t}^{-}(b)$ meet at some positive time, and $t=t_{a}^{+}(y)$ is the inverse of $y=\Phi_{t}^{+}(a)$, etc. 
Proof. It is standard to prove the local existence of Lipschitz solutions to (2.2) with smooth initial data. Now, we let $T^{*}$ be the life span of a Lipschitz solution to (2.2). It can be proved exactly as that in the proof of Lemma 6 of [18] that $\|R(t, \cdot)\|_{L^{\infty}}+\|S(t, \cdot)\|_{L^{\infty}}$ controls $T^{*}$; that is to say that $T^{*}<+\infty$ implies

$$
\lim _{t \rightarrow T^{*}}\left(\|R(t, \cdot)\|_{L^{\infty}}+\|S(t, \cdot)\|_{L^{\infty}}\right)=+\infty .
$$

Hence, in order to establish the global existence, it suffices to show that $\|R(t, \cdot)\|_{L^{\infty}}+\|S(t, \cdot)\|_{L^{\infty}}<+\infty$ for any $t<+\infty$.

We establish the estimates (2.3), (2.4), (2.6) for $0 \leqslant t<T^{*}$. Multiplying the first equation of (2.2) with $R(t, x)$, we find

$$
\begin{aligned}
\partial_{t} R^{2}-\partial_{x}\left(c(u) R^{2}\right)= & 2 \tilde{c}^{\prime}(u)^{+}\left\{-R\left(R^{2}-2 Q_{\varepsilon}(R)\right)+R^{2} S-R S^{2}\right\} \\
& +2 \tilde{c}^{\prime}(u)^{-}\left\{-R\left(R^{2}-2 Q_{-\varepsilon}(R)\right)+R^{2} S-R S^{2}\right\} .
\end{aligned}
$$

Similarly, we find

$$
\begin{aligned}
\partial_{t} S^{2}+\partial_{x}\left(c(u) S^{2}\right)= & 2 \tilde{c}^{\prime}(u)^{+}\left\{-S\left(S^{2}-2 Q_{\varepsilon}(S)\right)+R S^{2}-S R^{2}\right\} \\
& +2 \tilde{c}^{\prime}(u)^{-}\left\{-S\left(S^{2}-2 Q_{-\varepsilon}(S)\right)+R S^{2}-S R^{2}\right\} .
\end{aligned}
$$

Adding (2.8) and (2.9), we find

$$
\begin{aligned}
\partial_{t}\left(R^{2}+S^{2}\right)-\partial_{x}\left(c(u)\left(R^{2}-S^{2}\right)\right)= & -2 \tilde{c}^{\prime}(u)^{+}\left\{R\left(R^{2}-2 Q_{\varepsilon}(R)\right)+S\left(S^{2}-2 Q_{\varepsilon}(S)\right)\right\} \\
& -2 \tilde{c}^{\prime}(u)^{-}\left\{R\left(R^{2}-2 Q_{-\varepsilon}(R)\right)+S\left(S^{2}-2 Q_{-\varepsilon}(S)\right)\right\} \leqslant 0 .
\end{aligned}
$$

By integrating (2.10) over $\mathbb{R}$ with respect to $x$, we deduce (2.3) and (2.4). Integrating (2.10) over the characteristic cone $\Delta:=\left\{(t, x) \mid \Phi_{t}^{+}(a) \leqslant x \leqslant \Phi_{t}^{-}(b), 0 \leqslant t<T^{*}\right\}$, we deduce (2.6).

We establish $L^{\infty}$ bounds for $R$ and $S$. Fix a point $x \in \mathbb{R}$. We decompose the domain $\left\{t \in\left(0, T^{*}\right) \mid\right.$ $\left.R\left(t, \Phi_{t}^{-}(x)\right)<0\right\}$ into $\bigcup_{i=0}^{\infty}\left(t_{2 i}, t_{2 i+1}\right)$ in order to utilize the asymmetry in the chop-off of the equations. If $t_{0}=0$, then for $t \in\left[0, t_{1}\right)$, we have

$$
\frac{\mathrm{d}}{\mathrm{d} t} R\left(t, \Phi_{t}^{-}(x)\right) \geqslant\left\{-\tilde{c}^{\prime}(u)^{+} S^{2}+2 \tilde{c}^{\prime}(u)^{-} Q_{-\varepsilon}(R)\right\}\left(t, \Phi_{t}^{-}(x)\right) .
$$

But for $R \leqslant 0$, we have $Q_{-\varepsilon}(R) \leqslant-R / \varepsilon$. Thus

$$
\frac{\mathrm{d}}{\mathrm{d} t} R\left(t, \Phi_{t}^{-}(x)\right) \geqslant-\left\{\tilde{c}^{\prime}(u)^{+} S^{2}-\frac{M}{\varepsilon} R\right\}\left(t, \Phi_{t}^{-}(x)\right),
$$

which directly implies that

$$
R\left(t, \Phi_{t}^{-}(x)\right) \geqslant-\mathrm{e}^{M t / \varepsilon}\left(\left\|R_{0}\right\|_{L^{\infty}}+\frac{M_{1}}{8 C_{1}} \int\left(R_{0}^{2}+S_{0}^{2}\right)(x) \mathrm{d} x\right)
$$

for $t \in\left[0, t_{1}\right]$. For $i \geqslant 1$ (or $t_{0}>0$ ), as $R\left(t_{i}, \Phi_{t_{i}}^{-}(x)\right)=0$, we can repeat the above procedure to yield the same lower bound for $R\left(t, \Phi_{t}^{-}(x)\right)$ for $0 \leqslant t<T^{*}$. Since the solution $(R, S, u)$ is sufficiently smooth, this implies that

$$
R(t, x) \geqslant-\mathrm{e}^{M t / \varepsilon}\left(\left\|R_{0}\right\|_{L^{\infty}}+\frac{M_{1}}{8 C_{1}} \int\left(R_{0}^{2}+S_{0}^{2}\right)(x) \mathrm{d} x\right)
$$

for $0 \leqslant t<T^{*}$.

We have similarly obtain from (2.2) that

$$
\frac{\mathrm{d}}{\mathrm{d} t} R\left(t, \Phi_{t}^{-}(x)\right) \leqslant\left\{2 \tilde{c}^{\prime}(u)^{+} Q_{\varepsilon}(R)-\tilde{c}^{\prime}(u)^{-} S^{2}\right\}\left(t, \Phi_{t}^{-}(x)\right) .
$$


Thus, by repeating the proof of (2.14), we can prove

$$
R(t, x) \leqslant \mathrm{e}^{M t / \varepsilon}\left(\left\|R_{0}\right\|_{L^{\infty}}+\frac{M_{1}}{8 C_{1}} \int\left(R_{0}^{2}+S_{0}^{2}\right)(x) \mathrm{d} x\right) .
$$

Similarly, we can obtain an $\varepsilon$-dependent bound for $S$. This completes the proof of Lemma 2.1.

Now we can see from Lions-Aubin's Lemma, see Lemma 3 of [18] for details, that there exists a subsequence of the approximate solutions $\left\{u^{\varepsilon}\right\}$ which converges in the maximum norm on any compact domain of the upper half plane to a continuous function $u(t, x)$ :

$$
u^{\varepsilon} \rightarrow u(t, x) .
$$

We can use the continuity of $u(t, x)$ and $c^{\prime}(u)$ to obtain uniform estimates on $\left(R^{\varepsilon}, S^{\varepsilon}\right)$ in $L^{2+\alpha}$ at any point $(t, x)$ such that $c^{\prime}(u(t, x)) \neq 0$.

Lemma 2.2 (Local $L^{2+\alpha}$ estimate). Let $\left(R_{0}, S_{0}\right) \in L^{2}$. For solutions $\left\{\left(R^{\varepsilon}, S^{\varepsilon}, u^{\varepsilon}\right)\right\}_{\varepsilon>0}$ of $(2.2)$, there hold

$$
\begin{aligned}
& \frac{1-\alpha}{1+\alpha} \tilde{c}^{\prime}(u)(R-S)\left(R^{1+\alpha}-S^{1+\alpha}\right)+\tilde{c}^{\prime}(u) R^{\alpha} S^{\alpha}(R-S)\left(R^{1-\alpha}-S^{1-\alpha}\right) \\
& =\tilde{c}^{\prime}(u)^{+} R^{\alpha}\left(2 Q_{\varepsilon}(R)-R^{2}\right)+\tilde{c}^{\prime}(u)^{-} R^{\alpha}\left(2 Q_{-\varepsilon}(R)-R^{2}\right)+\tilde{c}^{\prime}(u)^{+} S^{\alpha}\left(2 Q_{\varepsilon}(S)-S^{2}\right) \\
& \quad+\tilde{c}^{\prime}(u)^{-} S^{\alpha}\left(2 Q_{-\varepsilon}(S)-S^{2}\right)-\frac{1}{1+\alpha}\left\{\partial_{t}\left(R^{1+\alpha}+S^{1+\alpha}\right)+\partial_{x}\left(c(u)\left(S^{1+\alpha}-R^{1+\alpha}\right)\right)\right\}
\end{aligned}
$$

and

$$
\iint_{\Omega}(R-S)^{2}\left(R^{\alpha}+S^{\alpha}\right) \mathrm{d} x \mathrm{~d} t \leqslant C_{\Omega, \alpha},
$$

where $\Omega$ is a small neighborhood of any point $(t, x)$ at which $c^{\prime}(u(t, x)) \neq 0, \alpha \in(0,1)$, and $C_{\Omega, \alpha}$ is independent of $\varepsilon$.

Proof. We assume without loss of generality that $\left(R_{0}, S_{0}\right) \in C_{c}^{\infty}(\mathbb{R})$. We take an $\alpha=d_{2} / d_{1} \in(0,1)$ where $d_{2}$ is an even positive integer and $d_{1}$ an odd positive integer. We then multiply the first equation of $(2.2)$ with $R^{\alpha}(t, x)$ to yield

$$
\begin{aligned}
& \frac{1}{1+\alpha}\left\{\partial_{t} R^{1+\alpha}-\partial_{x}\left(c(u) R^{1+\alpha}\right)\right\}+\frac{2}{1+\alpha} \tilde{c}^{\prime}(u)(R-S) R^{1+\alpha} \\
& \quad=\tilde{c}^{\prime}(u)^{+}\left(2 R^{\alpha} Q_{\varepsilon}(R)-R^{\alpha} S^{2}\right)+\tilde{c}^{\prime}(u)^{-}\left(2 R^{\alpha} Q_{-\varepsilon}(R)-R^{\alpha} S^{2}\right) .
\end{aligned}
$$

Splitting $2 /(1+\alpha)$ into $(1-\alpha) /(1+\alpha)+1$ and regrouping, we have

$$
\begin{aligned}
& \frac{1-\alpha}{1+\alpha} \tilde{c}^{\prime}(u)(R-S) R^{1+\alpha}+\tilde{c}^{\prime}(u)\left(R^{\alpha} S^{2}-S R^{1+\alpha}\right) \\
& \quad=\tilde{c}^{\prime}(u)^{+} R^{\alpha}\left(2 Q_{\varepsilon}(R)-R^{2}\right)+\tilde{c}^{\prime}(u)^{-} R^{\alpha}\left(2 Q_{-\varepsilon}(R)-R^{2}\right)-\frac{1}{1+\alpha}\left\{\partial_{t} R^{1+\alpha}-\partial_{x}\left(c(u) R^{1+\alpha}\right)\right\} .
\end{aligned}
$$

Similarly we have for $S$ :

$$
\begin{aligned}
& \frac{1-\alpha}{1+\alpha} \tilde{c}^{\prime}(u)(S-R) S^{1+\alpha}+\tilde{c}^{\prime}(u)\left(S^{\alpha} R^{2}-R S^{1+\alpha}\right) \\
& \quad=\tilde{c}^{\prime}(u)^{+} S^{\alpha}\left(2 Q_{\varepsilon}(S)-S^{2}\right)+\tilde{c}^{\prime}(u)^{-} S^{\alpha}\left(2 Q_{-\varepsilon}(S)-S^{2}\right)-\frac{1}{1+\alpha}\left\{\partial_{t} S^{1+\alpha}+\partial_{x}\left(c(u) S^{1+\alpha}\right)\right\} .
\end{aligned}
$$


Combining (2.21) and (2.22), we obtain (2.18).

Let $(t, x)$ be a point in the upper half plane so that $c^{\prime}(u(t, x)) \neq 0$. For definiteness, let us assume that $c^{\prime}(u(t, x)) \geqslant c_{0}$. Using the uniform convergence of $u^{\varepsilon}$, and the continuity of $c^{\prime}$, we can find a small neighborhood $\Omega_{1}$ of $(t, x)$ such that $c^{\prime}\left(u^{\varepsilon}(t, x)\right) \geqslant c_{0} / 2$ in $\Omega_{1}$ for all sufficiently small $\varepsilon$. Next we take an arbitrary open subset $\Omega$ of $\Omega_{1}$ with $\bar{\Omega} \subset \Omega_{1}$, and a cutoff function $\chi(t, x) \in C_{c}^{\infty}\left(\Omega_{1}\right)$ with $\chi(t, x)=1$ on $\bar{\Omega}$. Then multiplying $\chi$ to both sides of (2.18) and integrating the resulting identity over $\Omega$, we use integration by parts and the energy bounds in Lemma 2.1 to obtain

$$
\iint \chi\left[(R-S)\left(R^{1+\alpha}-S^{1+\alpha}\right)+R^{\alpha} S^{\alpha}(R-S)\left(R^{1-\alpha}-S^{1-\alpha}\right)\right] \mathrm{d} x \mathrm{~d} t \leqslant C_{\alpha, \chi} .
$$

Regrouping the integrand in (2.23), we obtain (2.19) by the definition of $\chi$.

To prove the precompactness of the approximate solutions $\left\{R^{\varepsilon}, S^{\varepsilon}\right\}$, we need the following type of entropy condition for $\left\{R^{\varepsilon}, S^{\varepsilon}\right\}$ :

Lemma 2.3. Let $\left(R_{0}, S_{0}\right) \in L^{2}(\mathbb{R})$. Let $t_{0}>0$ and $\left(t_{0}, x_{0}\right)$ be any point at which $c^{\prime}\left(u\left(t_{0}, x_{0}\right)\right) \neq 0$, then there exists a neighborhood $\mathcal{N}\left(t_{0}, x_{0}\right)$ of $\left(t_{0}, x_{0}\right)$ and some nonnegative constant $M\left(t_{0}, x_{0}\right)$ which is independent of $\varepsilon$, such that

$$
\operatorname{sign}\left(c^{\prime}\left(u^{\varepsilon}\right)\right) R^{\varepsilon}(t, x) \geqslant-M\left(t_{0}, x_{0}\right), \quad \operatorname{sign}\left(c^{\prime}\left(u^{\varepsilon}\right)\right) S^{\varepsilon}(t, x) \geqslant-M\left(t_{0}, x_{0}\right),
$$

hold for all $(t, x) \in \mathcal{N}\left(t_{0}, x_{0}\right)$.

Proof. Without loss of generality, we may assume that $c^{\prime}\left(u\left(t_{0}, x_{0}\right)\right)>0$, and denote $c^{\prime}\left(u\left(t_{0}, x_{0}\right)\right)$ by $c_{0}$. Then by (2.17), for small enough $\eta$, there is a ball $B_{\eta}\left(t_{0}, x_{0}\right)=\left\{(t, x)|| t-\left.t_{0}\right|^{2}+\left|x-x_{0}\right|^{2} \leqslant \eta^{2}\right\}$, such that

$$
c^{\prime}(u(t, x)) \geqslant \frac{c_{0}}{2}, \quad \text { and } \quad c^{\prime}\left(u^{\varepsilon}(t, x)\right) \geqslant \frac{c_{0}}{4} \quad \text { for }(t, x) \in B_{\eta}\left(t_{0}, x_{0}\right),
$$

for all sufficiently small $\varepsilon$. As $u^{\varepsilon}(t, x) \in L^{\infty}\left(\mathbb{R}^{+}, W^{1, \infty}(\mathbb{R})\right)$ (modify $\left(R_{0}, S_{0}\right)$ if necessary), the plus and minus characteristics $\Phi_{t}^{\varepsilon, \pm}(y)$ defined by

$$
\left\{\begin{array}{l}
\frac{\mathrm{d}}{\mathrm{d} t} \Phi_{t}^{\varepsilon, \pm}(y)= \pm c\left(u^{\varepsilon}\left(t, \Phi_{t}^{\varepsilon, \pm}(y)\right)\right), \\
\left.\Phi_{t}^{\varepsilon, \pm}(y)\right|_{t=0}=y,
\end{array}\right.
$$

can pass through any point in the upper plane.

Fix a $y \in \mathbb{R}$. Assume that $\left[t_{1}, t_{2}\right]$ is the largest time interval such that $\left(t, \Phi_{t}^{\varepsilon,-}(y)\right) \in B_{\eta}\left(t_{0}, x_{0}\right)$ for $t \in\left[t_{1}, t_{2}\right]$, and $\left[t_{3}, t_{4}\right]$ is the largest time interval such that $\left(t, \Phi_{t}^{\varepsilon,-}(y)\right) \in B_{\eta / 2}\left(t_{0}, x_{0}\right)$ for $t \in\left[t_{3}, t_{4}\right]$. By (1.9) and (2.25), we have

$$
t_{3}-t_{1} \geqslant \delta \eta
$$

for some positive constant $\delta$. By (2.6), we can find some positive constant $K_{1}$, which depends only on the $L^{2}$ norm of $\left(R_{0}, S_{0}\right)$, such that

$$
\int_{0}^{\infty}\left(\left|\tilde{c}^{\prime}\left(u^{\varepsilon}\right)\right|\left(S^{\varepsilon}\right)^{2}\right)\left(s, \Phi_{s}^{\varepsilon,-}(y)\right) \mathrm{d} s \leqslant K_{1} .
$$

Let us take $K_{2}=2 \sqrt{K_{1} /\left(c_{0} \delta \eta\right)}$. Then if $R^{\varepsilon}\left(t_{1}, \Phi_{t_{1}}^{\varepsilon,-}(y)\right) \geqslant-K_{2}$, by (2.2) and (2.26), we have

$$
\frac{\mathrm{d} R^{\varepsilon}\left(t, \Phi_{t}^{\varepsilon,-}(y)\right)}{\mathrm{d} t} \geqslant-\tilde{c}^{\prime}\left(u^{\varepsilon}\right)\left(S^{\epsilon}\right)^{2}\left(t, \Phi_{t}^{\varepsilon,-}(y)\right), \quad t \in\left[t_{1}, t_{2}\right] .
$$


Integrating the above inequality over $\left[t_{1}, t\right]$ with $t \leqslant t_{2}$, we find by (2.28) that

$$
R^{\varepsilon}\left(t, \Phi_{t}^{\varepsilon,-}(y)\right) \geqslant R^{\varepsilon}\left(t_{1}, \Phi_{t_{1}}^{\varepsilon,-}(y)\right)-\int_{t_{1}}^{t} \tilde{c}^{\prime}\left(u^{\varepsilon}\right)\left(S^{\epsilon}\right)^{2}\left(s, \Phi_{s}^{\varepsilon,-}(y)\right) \mathrm{d} s \geqslant-K_{2}-K_{1} .
$$

Otherwise, let us assume

$$
R^{\varepsilon}\left(t, \Phi_{t}^{\varepsilon,-}(y)\right) \leqslant-K_{2} \quad \text { for } t \in\left[t_{1}, \tau\right], \tau \leqslant t_{2},
$$

and $R^{\varepsilon}\left(\tau, \Phi_{\tau}^{\varepsilon,-}(y)\right)=-K_{2}$. Then by the proof of (2.29), we have

$$
R^{\varepsilon}\left(t, \Phi_{t}^{\varepsilon,-}(y)\right) \geqslant-K_{2}-K_{1}, \quad t_{2} \geqslant t \geqslant \tau .
$$

And by (2.1), (2.2) and (2.26), there holds

$$
\frac{\mathrm{d} R^{\varepsilon}\left(t, \Phi_{t}^{\varepsilon,-}(y)\right)}{\mathrm{d} t}=\tilde{c}^{\prime}\left(u^{\epsilon}\right)\left(\left(R^{\varepsilon}\right)^{2}-\left(S^{\varepsilon}\right)^{2}\right)\left(t, \Phi_{t}^{\varepsilon,-}(y)\right), \quad t \in\left[t_{1}, \tau\right] .
$$

Let us divide the equation by $\left(R^{\varepsilon}\right)^{2}$ and then integrate it over $\left[t_{1}, t\right]$ to obtain

$$
\begin{aligned}
\frac{1}{R^{\varepsilon}\left(t, \Phi_{t}^{\varepsilon,-}(y)\right)} & =\frac{1}{R^{\varepsilon}\left(t_{1}, \Phi_{t_{1}}^{\varepsilon,-}(y)\right)}-\int_{t_{1}}^{t} \tilde{c}^{\prime}\left(u^{\varepsilon}\left(s, \Phi_{s}^{\varepsilon,-}(y)\right)\right) \mathrm{d} s+\int_{t_{1}}^{t} \tilde{c}^{\prime}\left(u^{\varepsilon}\right)\left(\frac{S^{\varepsilon}}{R^{\varepsilon}}\right)^{2}\left(s, \Phi_{s}^{\varepsilon,-}(y)\right) \mathrm{d} s \\
& \leqslant-\frac{c_{0}}{4}\left(t-t_{1}\right)+\frac{K_{1}}{K_{2}^{2}} \leqslant-\frac{c_{0} \delta \eta}{8}
\end{aligned}
$$

if $t_{1}+\delta \eta \leqslant t \leqslant \tau$, where we have used (2.25), (2.28), and (2.30). Thus we have

$$
R^{\varepsilon}\left(t, \Phi_{t}^{\varepsilon,-}(y)\right) \geqslant-\frac{8}{c_{0} \delta \eta}, \quad t \in\left[t_{1}+\delta \eta, \tau\right] .
$$

Let us take $M\left(t_{0}, x_{0}\right)=\max \left(K_{1}+K_{2}, 8 /\left(c_{0} \delta \eta\right)\right)$. By summing up (2.29), (2.31) and (2.33), we have

$$
R^{\varepsilon}\left(t, \Phi_{t}^{\varepsilon,-}(y)\right) \geqslant-M\left(t_{0}, x_{0}\right), \quad t \in\left[t_{1}+\delta \eta, t_{2}\right] .
$$

This together with (2.27) imply that

$$
R^{\varepsilon}(t, x) \geqslant-M\left(t_{0}, x_{0}\right), \quad \text { for }(t, x) \in B_{\eta / 2}\left(t_{0}, x_{0}\right) .
$$

Similarly, we can prove

$$
S^{\varepsilon}(t, x) \geqslant-M\left(t_{0}, x_{0}\right), \quad \text { for }(t, x) \in B_{\eta / 2}\left(t_{0}, x_{0}\right) .
$$

Combining (2.35) and (2.36), and taking $\mathcal{N}\left(t_{0}, x_{0}\right)=B_{\eta / 2}\left(t_{0}, x_{0}\right)$, we obtain (2.24).

We are going to assume the particular conditions $c(\cdot) \geqslant 0$ and $c^{\prime}\left(u_{0}(\cdot)\right)>0$ in the next lemma. The condition $c^{\prime}\left(u_{0}(\cdot)\right)>0$ drives the solution $(R, S)$ away from possible negative infinity in a thin initial layer, while the condition $c(\cdot) \geqslant 0$ will maintain the lower bounds for the rest of the time. We note that Sobolev embedding gives a positive constant $L$ such that $\left\|u_{0}\right\|_{L^{\infty}} \leqslant L$ when $u_{0} \in H^{1}(\mathbb{R})$.

Lemma 2.4. Let $c^{\prime}(\cdot) \geqslant 0, c^{\prime}\left(u_{0}(\cdot)\right)>0$, and $\left(R_{0}, S_{0}\right) \in L^{2}(\mathbb{R})$, let $t_{0}>0$ be any sufficiently small positive number. Then for any $\bar{x} \in \mathbb{R}$ and any $\bar{t}>t_{0}$, there exist a neighbourhood $\mathcal{N}(\bar{t}, \bar{x})$ and some positive constant $\bar{M}$, which depends only on the $L^{2}$ norm of $\left(R_{0}, S_{0}\right), t_{0}$, and $c^{\prime}\left(u_{0}\right)$, such that

$$
R^{\epsilon}(t, x) \geqslant-\bar{M}, \quad S^{\epsilon}(t, x) \geqslant-\bar{M}
$$

for all $(t, x) \in \mathcal{N}(\bar{t}, \bar{x})$. 
Proof. For any compact subset $C_{1}$ of $\mathbb{R}$, there exists a constant $c_{0}>0$ such that

$$
c^{\prime}\left(u_{0}(x)\right) \geqslant c_{0}, \quad \forall x \in C_{1} .
$$

Thus by (2.17), we find that for sufficiently small $t_{0}$, there holds

$$
c^{\prime}\left(u\left(t_{0}, x\right)\right) \geqslant \frac{c_{0}}{2}, \quad x \in C_{1} .
$$

Hence in particular, (2.24) and its proof imply that

$$
R^{\epsilon}\left(t_{0}, x\right) \geqslant-M_{0}\left(t_{0}\right), \quad x \in C_{1},
$$

where $M_{0}\left(t_{0}\right)$ depends only on the $L^{2}$ norm of $\left(R_{0}, S_{0}\right), t_{0}$, and $c_{0}$. Then, (2.40) and a proof similar to that of (2.29) imply that

$$
R^{\epsilon}\left(t, \Phi_{t}^{\epsilon,-}(y)\right) \geqslant-M_{0}\left(t_{0}\right)-K_{1}=: M_{1}, \quad t \geqslant t_{0}, y \in C_{1} .
$$

Now let us take a countable compact subsets $C_{i}$ such that $C_{i} \subset C_{i+1}$ and $\mathbb{R}=\bigcup_{i=1}^{\infty} C_{i}$. Then by a proof similar to that of (2.41), we can find for each $i$ a constant $M_{i}\left(t_{0}\right)>0$, which depends only on the $L^{2}$ norm of $\left(R_{0}, S_{0}\right)$ and $c_{0}$, such that

$$
R^{\epsilon}\left(t, \Phi_{t}^{\epsilon,-}(y)\right) \geqslant-M_{i}\left(t_{0}\right), \quad y \in C_{i}, t \geqslant t_{0} .
$$

On the other hand, as $\left(t, \Phi_{t}^{\epsilon,-}(y)\right)$ can pass through any point in the upper half plane, for any $(\bar{t}, \bar{x}) \in\left(t_{0}, \infty\right) \times \mathbb{R}$, we can always find a neighbourhood $\mathcal{N}(\bar{t}, \bar{x})$ such that every point of $\mathcal{N}(\bar{t}, \bar{x})$ can be reached by a characteristic curve $\left(t, \Phi_{t}^{\epsilon,-}(y)\right)$ starting from $y \in C_{i}$ for some fixed $i$. Hence by (2.42), we have

$$
R^{\epsilon}(t, x) \geqslant-M_{i}\left(t_{0}\right), \quad(t, x) \in \mathcal{N}(\bar{t}, \bar{x})
$$

Similar argument for $S^{\epsilon}$ yields a similar inequality as (2.43) for $S^{\epsilon}$. The proof of the lemma is complete.

\section{Precompactness}

Let $\left(R_{0}, S_{0}\right) \in L^{2}(\mathbb{R})$. Let $j_{\varepsilon}(x)$ be the standard Friedrichs' mollifier, and $\chi_{\epsilon}(x)=\chi\left(\frac{x}{\epsilon}\right)$ with $\chi(x) \in C_{c}^{\infty}(\mathbb{R})$ and $\chi(x)=1$ around $x=0$. We denote $R_{0}^{\varepsilon}=\left(R_{0} \chi_{\epsilon}\right) * j_{\varepsilon}$ and $S_{0}^{\varepsilon}=\left(S_{0} \chi_{\epsilon}\right) * j_{\varepsilon}$. Then by Lemma 2.1, problem (2.2) has a global smooth solution $\left(R^{\varepsilon}, S^{\varepsilon}, u^{\varepsilon}\right)$ with the initial data $\left(R_{0}^{\varepsilon}, S_{0}^{\varepsilon}\right)$. Moreover, we have

$$
\int\left(\left(R^{\varepsilon}\right)^{2}+\left(S^{\varepsilon}\right)^{2}\right)(t, x) \mathrm{d} x \leqslant \int\left(R_{0}^{2}+S_{0}^{2}\right)(x) \mathrm{d} x \quad(t \geqslant 0) .
$$

We shall also use energy estimate (2.6) and (2.19) in this new setting.

We establish the precompactness of $\left\{\left(R^{\varepsilon}, S^{\varepsilon}, u^{\varepsilon}\right)(t, x)\right\}$ in this section.

Next, for the convenience of the reader, we recall the following lemma (see Lemmas 9-10 of [18]).

Lemma 3.1 (Time-distinguished Young measure [17,15,11,3]). There exist a subsequence of the solution sequence $\left\{R^{\varepsilon}(t, x), S^{\varepsilon}(t, x)\right\}$, which we still denote by $\left\{R^{\varepsilon}(t, x), S^{\varepsilon}(t, x)\right\}$ for convenience, and three families of Young measures $v_{t x}^{1}(\xi)$ on $\mathbb{R}, v_{t x}^{2}(\eta)$ on $\mathbb{R}$, and $\mu_{t x}(\xi, \eta)$ on $\mathbb{R}^{2}$, such that for all continuous functions $f(\lambda) \in C_{c}^{\infty}(\mathbb{R})$, $\psi(x) \in C_{c}^{\infty}(\mathbb{R}), g(\xi, \eta) \in C^{\infty}\left(\mathbb{R}^{2}\right)$ with $g(\xi, \eta)=o\left((|\xi|+|\eta|)^{p}\right)$ as $|\xi|+|\eta| \rightarrow \infty$ for some $p<2$, and $\varphi(t, x) \in$ $C_{c}^{\infty}\left(\mathbb{R}^{+} \times \mathbb{R}\right)$, there hold

$$
\begin{aligned}
& \lim _{\varepsilon \rightarrow 0} \int_{\mathbb{R}} f\left(R^{\varepsilon}(t, x)\right) \psi(x) \mathrm{d} x=\iint_{\mathbb{R} \times \mathbb{R}} f(\xi) \psi(x) \mathrm{d} v_{t x}^{1}(\xi) \mathrm{d} x, \\
& \lim _{\varepsilon \rightarrow 0} \int_{\mathbb{R}} f\left(S^{\varepsilon}(t, x)\right) \psi(x) \mathrm{d} x=\iint_{\mathbb{R} \times \mathbb{R}} f(\eta) \psi(x) \mathrm{d} v_{t x}^{2}(\eta) \mathrm{d} x,
\end{aligned}
$$


uniformly in every compact subset of $[0, \infty)$, and

$$
\lim _{\varepsilon \rightarrow 0} \int_{0}^{\infty} \int_{\mathbb{R}} g\left(R^{\varepsilon}(t, x), S^{\varepsilon}(t, x)\right) \varphi(t, x) \mathrm{d} x \mathrm{~d} t=\int_{0}^{\infty} \int_{\mathbb{R}} \int_{\mathbb{R} \times \mathbb{R}} g(\xi, \eta) \varphi(t, x) \mathrm{d} \mu_{t x}(\xi, \eta) \mathrm{d} x \mathrm{~d} t .
$$

Moreover,

$$
\begin{aligned}
& t \in[0, \infty) \mapsto \int_{\mathbb{R} \times \mathbb{R}} f(\xi) \psi(x) \mathrm{d} v_{t x}^{1}(\xi) \mathrm{d} x \text { is continuous, } \\
& t \in[0, \infty) \mapsto \iint_{\mathbb{R} \times \mathbb{R}} f(\eta) \psi(x) \mathrm{d} v_{t x}^{2}(\eta) \mathrm{d} x \text { is continuous, }
\end{aligned}
$$

and

$$
\mu_{t x}(\xi, \eta)=v_{t x}^{1}(\xi) \otimes v_{t x}^{2}(\eta)
$$

Furthermore, by Proposition 3.1.3 of [11] and Lemma 3.1, we find that

$$
\begin{aligned}
& \xi \in L^{\infty}\left(\mathbb{R}^{+}, L^{2}\left(\mathbb{R} \times \mathbb{R}, \mathrm{d} x \otimes \mathrm{d} v_{t x}^{1}(\xi)\right)\right), \\
& \eta \in L^{\infty}\left(\mathbb{R}^{+}, L^{2}\left(\mathbb{R} \times \mathbb{R}, \mathrm{d} x \otimes \mathrm{d} v_{t x}^{2}(\eta)\right)\right) .
\end{aligned}
$$

We remark that (3.5) implies directly that

$$
R^{\epsilon} S^{\epsilon} \rightarrow R S \text { as } \epsilon \rightarrow 0
$$

in the sense of distributions. Glassey, Hunter and Zheng [6] have derived (3.7) by applying the div-curl lemma for a sequence of energy conservative weak solutions $\left\{u^{\varepsilon}(t, x)\right\}$ of $(1.1)$, assuming that $\left\{u^{\varepsilon}(t, x)\right\}$ is uniformly bounded in $W^{1, p}\left(\mathbb{R}^{+} \times \mathbb{R}\right)$ for some $p>2$. Here we have provided a local version of this uniform estimate, but we do not need the energy conservation assumption for the approximate solutions.

We shall use the notation

$$
\overline{g(R, S)}=\int_{\mathbb{R}} g(\xi, \eta) \mathrm{d} \mu_{t x}(\xi, \eta) .
$$

Thus, $(\bar{R}, \bar{S})$ represents the weak star limit of $\left\{R^{\varepsilon}, S^{\varepsilon}\right\}$ in $L^{\infty}\left(\mathbb{R}^{+}, L^{2}(\mathbb{R})\right)$ or the weak limit in $L^{2}((0, T) \times \mathbb{R})$ for all $T<\infty$.

With the above preparation, we can now establish the precompactness of $\left\{R^{\varepsilon}, S^{\varepsilon}\right\}$.

Lemma 3.2 (Precompactness of $\left.\left\{\left(R^{\varepsilon}, S^{\varepsilon}\right)\right\}\right)$. Let $c^{\prime}(\cdot) \geqslant 0, c^{\prime}\left(u_{0}(\cdot)\right)>0$, and $\left(R_{0}(x), S_{0}(x)\right) \in L^{2}(\mathbb{R})$. Then $v_{t x}^{1}(\xi)=\delta_{\bar{R}(t, x)}(\xi)$ and $v_{t x}^{2}(\eta)=\delta_{\bar{S}(t, x)}(\eta)$.

Proof. Since the proof of $v_{t x}^{1}(\xi)=\delta_{\bar{R}(t, x)}(\xi)$ is the same as that of $v_{t x}^{2}(\eta)=\delta_{\bar{S}(t, x)}(\eta)$, we present only the proof for the former.

The idea is to derive an evolution equation (inequality) for the quantity $\overline{R^{2}}-\bar{R}^{2}$, so that it is zero for all positive time if it is zero at time zero which is true in our case. In the derivation of the evolution equation we need to cut off desired multipliers and mollify various equations that are true only in the weak sense. We will present the proof of Theorem 1.1 as general as we can. The assumptions that $c^{\prime}(\cdot) \geqslant 0$ and $c^{\prime}\left(u_{0}(\cdot)\right)>0$ will only be used in step 6 . 
Step 1. Derivation of the equation for $\bar{R}$.

We write the first equation of (2.2) in the form

$$
\partial_{t} R^{\varepsilon}-\partial_{x}\left(c\left(u^{\varepsilon}\right) R^{\varepsilon}\right)=-\tilde{c}^{\prime}\left(u^{\varepsilon}\right)\left(R^{\varepsilon}-S^{\varepsilon}\right)^{2}-\tilde{c}^{\prime+}\left[\left(R^{\varepsilon}\right)^{2}-2 Q_{\varepsilon}\left(R^{\varepsilon}\right)\right]-\tilde{c}^{\prime-}\left[\left(R^{\varepsilon}\right)^{2}-2 Q_{-\varepsilon}\left(R^{\varepsilon}\right)\right] .
$$

We claim that there holds in the sense of distributions

$$
\tilde{c}^{\prime}\left(u^{\varepsilon}\right)\left(R^{\varepsilon}-S^{\varepsilon}\right)^{2} \rightarrow \tilde{c}^{\prime}(u) \iint_{\mathbb{R}^{2}}(\xi-\eta)^{2} \mathrm{~d} \mu_{t x}(\xi, \eta)=\tilde{c}^{\prime}(u)\left(\overline{R^{2}}+\overline{S^{2}}-2 \bar{R} \bar{S}\right) .
$$

The claim will follow from Lemma 3.1 and estimate (2.19). At a point $(t, x)$ where $c^{\prime}(u(t, x)) \neq 0$, we assume without loss of generality that $c^{\prime}(u(t, x))>0$. We take a neighborhood $\Omega$ of $(t, x)$ where $c^{\prime}\left(u^{\varepsilon}(t, x)\right)>M_{0}>0$ for all small $\varepsilon>0$. We take a smooth cut-off function $\psi(\xi)$ with $\psi(\xi)=1$ for $|\xi| \leqslant 1$ and $\operatorname{supp} \psi \subset\{\xi|| \xi \mid \leqslant 2\}$. Then, by (2.19), we have

$$
\iint_{\Omega}\left|R^{\varepsilon}\left(R^{\varepsilon}-S^{\varepsilon}\right)\right|\left(1-\psi\left(\frac{R^{\epsilon}}{k}\right)\right) \mathrm{d} x \mathrm{~d} t \leqslant C \operatorname{meas}\left\{(t, x) \in \Omega \mid R^{\varepsilon} \geqslant k\right\}^{1 / p} \leqslant C k^{-2 / p},
$$

where $\frac{1}{p}=\frac{\alpha}{2(2+\alpha)}$ for any $0<\alpha<1$. While by Lemma 3.1, for any test function $\phi(t, x) \in C_{c}^{\infty}(\Omega)$, there holds

$$
\lim _{\varepsilon \rightarrow 0} \int_{0}^{\infty} \int_{\mathbb{R}} \phi \tilde{c}^{\prime}\left(u^{\varepsilon}\right) R^{\varepsilon}\left(R^{\varepsilon}-S^{\varepsilon}\right) \psi\left(\frac{R^{\epsilon}}{k}\right) \mathrm{d} x \mathrm{~d} t=\iint_{\mathbb{R}^{+} \times \mathbb{R}} \phi \tilde{c}^{\prime}(u) \iint_{\mathbb{R}^{2}} \xi(\xi-\eta) \psi\left(\frac{\xi}{k}\right) \mathrm{d} \mu_{t, x}(\xi, \eta) \mathrm{d} x \mathrm{~d} t .
$$

By summing up (3.6), (3.10), (3.11) and Lebesgue Dominated Convergence Theorem as $k \rightarrow \infty$, we find

$$
\lim _{\varepsilon \rightarrow 0} \iint_{0}^{\infty} \phi \tilde{c}^{\prime}\left(u^{\varepsilon}\right) R^{\varepsilon}\left(R^{\varepsilon}-S^{\varepsilon}\right) \mathrm{d} x \mathrm{~d} t=\iint_{\mathbb{R}^{+} \times \mathbb{R}} \phi \tilde{c}^{\prime}(u) \iint_{\mathbb{R}^{2}} \xi(\xi-\eta) \mathrm{d} \mu_{t, x}(\xi, \eta) \mathrm{d} x \mathrm{~d} t .
$$

A similar proof yields that

$$
\lim _{\varepsilon \rightarrow 0} \iint_{0}^{\infty} \phi \tilde{c}^{\prime}\left(u^{\varepsilon}\right) S^{\varepsilon}\left(R^{\varepsilon}-S^{\varepsilon}\right) \mathrm{d} x \mathrm{~d} t=\iint_{\mathbb{R}^{+} \times \mathbb{R}} \phi \tilde{c}^{\prime}(u) \iint_{\mathbb{R}^{2}} \eta(\xi-\eta) \mathrm{d} \mu_{t, x}(\xi, \eta) \mathrm{d} x \mathrm{~d} t .
$$

It follows from (3.12) and (3.13) that Claim (3.9) holds for all test functions $\varphi \in C_{c}^{\infty}(\Omega(t, x))$.

Now let us denote $\mathcal{G}:=\left\{(t, x), c^{\prime}(u(t, x)) \neq 0\right\}$. For any $(t, x) \in \mathcal{G}$, let us denote $\Omega(t, x)$ a neighborhood of $(t, x)$ such that (3.9) holds for all test functions $\varphi \in C_{c}^{\infty}(\Omega(t, x))$. Then for any test function $\varphi(t, x) \in C_{c}^{\infty}\left(\mathbb{R}^{+} \times \mathbb{R}\right)$ and any $a>0$, as $\mathcal{H}=\operatorname{supp} \varphi \cap\left\{(t, x)\left|c^{\prime}(u(t, x))\right| \geqslant a\right\}$ is a compact subset of $\mathcal{G}$, it can be covered by a finite union of $\Omega\left(t_{i}, x_{i}\right), i=1, \ldots, N$. By basic topology, we can take a partition of unity subordinate to $\bigcup_{i=1}^{N} \Omega\left(t_{i}, x_{i}\right)$, i.e., there are $\phi_{i}(t, x) \in C_{c}^{\infty}\left(\Omega\left(t_{i}, x_{i}\right)\right)$, such that $\sum_{i=1}^{N} \phi_{i}(t, x)=1$ for $(t, x) \in \mathcal{H}$. Next, let us decompose the left-hand side of (3.9) in the following form:

$$
\iint \varphi \tilde{c}^{\prime}\left(u^{\varepsilon}\right)\left(R^{\varepsilon}-S^{\varepsilon}\right)^{2} \mathrm{~d} x \mathrm{~d} t=\iint\left(1_{\left|c^{\prime}(u)\right| \geqslant a}+1_{\left|c^{\prime}(u)\right| \leqslant a}\right) \varphi \tilde{c}^{\prime}\left(u^{\varepsilon}\right)\left(R^{\varepsilon}-S^{\varepsilon}\right)^{2} \mathrm{~d} x \mathrm{~d} t,
$$

and

$$
\begin{gathered}
\lim _{\varepsilon \rightarrow 0} \iint 1_{\left|c^{\prime}(u)\right| \geqslant a} \varphi \tilde{c}^{\prime}\left(u^{\varepsilon}\right)\left(R^{\varepsilon}-S^{\varepsilon}\right)^{2} \mathrm{~d} x \mathrm{~d} t=\lim _{\varepsilon \rightarrow 0} \sum_{i=1}^{N} \iint 1_{\left|c^{\prime}(u)\right| \geqslant a} \varphi \phi_{i} \tilde{c}^{\prime}\left(u^{\varepsilon}\right)\left(R^{\varepsilon}-S^{\varepsilon}\right)^{2} \mathrm{~d} x \mathrm{~d} t \\
=\sum_{i=1}^{N} \iint 1_{\left|c^{\prime}(u)\right| \geqslant a} \varphi \phi_{i} \tilde{c}^{\prime}(u) \overline{(R-S)^{2}} \mathrm{~d} x \mathrm{~d} t=\iint 1_{\left|c^{\prime}(u)\right| \geqslant a} \varphi \tilde{c}^{\prime}(u) \overline{(R-S)^{2}} \mathrm{~d} x \mathrm{~d} t,
\end{gathered}
$$


and trivially by (3.1), we have

$$
\left|\iint 1_{\left|c^{\prime}(u)\right| \leqslant a} \varphi \tilde{c}^{\prime}\left(u^{\varepsilon}\right)\left(R^{\varepsilon}-S^{\varepsilon}\right)^{2} \mathrm{~d} x \mathrm{~d} t\right| \leqslant C a .
$$

By summing up (3.14)-(3.16), we take $\varepsilon \rightarrow 0$ then $a \rightarrow 0$ in (3.14), we prove claim (3.9).

Now, from (2.4), we find easily that

$$
\lim _{\varepsilon \rightarrow 0} \int_{0}^{T} \int_{\mathbb{R}} \tilde{c}^{\prime \pm}\left(u^{\varepsilon}\right)\left[\left(R^{\varepsilon}\right)^{2}-2 Q_{ \pm \varepsilon}\left(R^{\varepsilon}\right)\right] \mathrm{d} x \mathrm{~d} t=0 .
$$

Thus, again by (3.5)-(3.6), (3.9), and (3.17), we have

$$
\partial_{t} \bar{R}-\partial_{x}(c(u) \bar{R})=-\tilde{c}^{\prime}(u)\left(\overline{R^{2}}-2 \bar{R} \bar{S}+\overline{S^{2}}\right) .
$$

Step 2. Cut-off of $\left(R^{\varepsilon}\right)^{2}$.

Similarly to [19], let us define for $\lambda>0$

$$
T_{\lambda}(\xi)=\left\{\begin{array}{ll}
\xi, & |\xi| \leqslant \lambda, \\
\lambda, & \xi \geqslant \lambda, \\
-\lambda, & \xi \leqslant-\lambda,
\end{array} \quad S_{\lambda}(\xi)= \begin{cases}\frac{1}{2} \xi^{2}, & |\xi| \leqslant \lambda, \\
\lambda\left(\xi-\frac{\lambda}{2}\right), & \xi \geqslant \lambda, \\
-\lambda\left(\xi+\frac{\lambda}{2}\right), & \xi \leqslant-\lambda .\end{cases}\right.
$$

We multiply the first equation of (2.2) with $T_{\lambda}\left(R^{\varepsilon}\right)$ to obtain

$$
\begin{aligned}
\partial_{t} S_{\lambda}\left(R^{\varepsilon}\right)-\partial_{x}\left(c\left(u^{\varepsilon}\right) S_{\lambda}\left(R^{\varepsilon}\right)\right)= & -2 \tilde{c}^{\prime}\left(u^{\varepsilon}\right)\left(R^{\varepsilon}-S^{\varepsilon}\right) S_{\lambda}\left(R^{\varepsilon}\right)+\tilde{c}^{\prime}\left(u^{\varepsilon}\right)^{+} T_{\lambda}\left(R^{\varepsilon}\right)\left(2 Q_{\varepsilon}\left(R^{\varepsilon}\right)-\left(S^{\varepsilon}\right)^{2}\right) \\
& +\tilde{c}^{\prime}\left(u^{\varepsilon}\right)^{-} T_{\lambda}\left(R^{\varepsilon}\right)\left(2 Q_{-\varepsilon}\left(R^{\varepsilon}\right)-\left(S^{\varepsilon}\right)^{2}\right) .
\end{aligned}
$$

By Lemma 3.1, a similar proof of (3.9) and (3.17), we find that

$$
\begin{aligned}
& \tilde{c}^{\prime}\left(u^{\varepsilon}\right)\left(R^{\varepsilon}-S^{\varepsilon}\right) S_{\lambda}\left(R^{\varepsilon}\right) \rightarrow \tilde{c}^{\prime}(u) \overline{(R-S) S_{\lambda}(R)}, \\
& \tilde{c}^{\prime}\left(u^{\varepsilon}\right)^{+} T_{\lambda}\left(R^{\varepsilon}\right)\left(2 Q_{\varepsilon}\left(R^{\varepsilon}\right)-\left(S^{\varepsilon}\right)^{2}\right)+\tilde{c}^{\prime}\left(u^{\varepsilon}\right)^{-} T_{\lambda}\left(R^{\varepsilon}\right)\left(2 Q_{-\varepsilon}\left(R^{\varepsilon}\right)-\left(S^{\varepsilon}\right)^{2}\right) \rightarrow \tilde{c}^{\prime}(u) \overline{T_{\lambda}(R)\left(R^{2}-S^{2}\right) .}
\end{aligned}
$$

Taking $\varepsilon \rightarrow 0$ in (3.19), we obtain

$$
\partial_{t} \overline{S_{\lambda}(R)}-\partial_{x}\left(c(u) \overline{S_{\lambda}(R)}\right)=\tilde{c}^{\prime}(u)\left\{-2 \overline{R S_{\lambda}(R)}+\overline{T_{\lambda}(R) R^{2}}+2 \bar{S} \overline{S_{\lambda}(R)}-\overline{T_{\lambda}(R)} \overline{S^{2}}\right\} .
$$

Step 3. Cut-off of $\bar{R}^{2}$

Convolving (3.18) with the standard Friedrichs' mollifier $j_{\varepsilon}$, we find

$$
\partial_{t} \bar{R}^{\varepsilon}-\partial_{x}\left(c(u) \bar{R}^{\varepsilon}\right)=-\left(\tilde{c}^{\prime}(u) \overline{(R-S)^{2}}\right) * j_{\varepsilon}+\gamma_{\varepsilon},
$$

where $\bar{R}^{\varepsilon}=\int_{\mathbb{R}} \bar{R}(t, y) j_{\varepsilon}(x-y) \mathrm{d} y$ and $\gamma_{\varepsilon}=j_{\varepsilon} * \partial_{x}(c(u) \bar{R})-\partial_{x}\left(c(u) \bar{R}_{\varepsilon}\right)$. By DiPerna-Lions renormalization Lemma 2.3 of Lions [12] and Lebesgue Dominated Convergence Theorem in the time direction, we have $\gamma_{\varepsilon} \rightarrow 0$ in $L_{\text {loc }}^{1}\left(\mathbb{R}^{+} \times \mathbb{R}\right)$ (or see Lemma 2.1 of [1]). Again, we multiply (3.21) with $T_{\lambda}\left(\bar{R}^{\varepsilon}\right)$ to find

$$
\begin{aligned}
& \partial_{t} S_{\lambda}\left(\bar{R}^{\varepsilon}\right)-\partial_{x}\left(c(u) S_{\lambda}\left(\bar{R}^{\varepsilon}\right)\right) \\
& \quad=-2 \tilde{c}^{\prime}(u)(\bar{R}-\bar{S}) S_{\lambda}\left(\bar{R}^{\varepsilon}\right)+\left[-\left(\tilde{c}^{\prime}(u) \overline{(R-S)^{2}}\right) * j_{\varepsilon}+\gamma_{\varepsilon}+2 \tilde{c}^{\prime}(u)(\bar{R}-\bar{S}) \bar{R}^{\varepsilon}\right] T_{\lambda}\left(\bar{R}^{\varepsilon}\right) .
\end{aligned}
$$


Taking $\varepsilon \rightarrow 0$ in (3.22), we find

$$
\partial_{t} S_{\lambda}(\bar{R})-\partial_{x}\left(c(u) S_{\lambda}(\bar{R})\right)=\left[-\tilde{c}^{\prime}(u) \overline{(R-S)^{2}}+2 \tilde{c}^{\prime}(u)(\bar{R}-\bar{S}) \bar{R}\right] T_{\lambda}(\bar{R})-2 \tilde{c}^{\prime}(u)(\bar{R}-\bar{S}) S_{\lambda}(\bar{R}) .
$$

Or

$$
\begin{aligned}
& \partial_{t} S_{\lambda}(\bar{R})-\partial_{x}\left(c(u) S_{\lambda}(\bar{R})\right) \\
& \quad=\tilde{c}^{\prime}(u)\left\{-2 \bar{R} S_{\lambda}(\bar{R})+T_{\lambda}(\bar{R}) \bar{R}^{2}+2 \bar{S} S_{\lambda}(\bar{R})-T_{\lambda}(\bar{R}) \overline{S^{2}}-T_{\lambda}(\bar{R})\left(\overline{R^{2}}-\bar{R}^{2}\right)\right\} .
\end{aligned}
$$

Step 4. Evolution equation for " $\overline{R^{2}}-\bar{R}^{2}$ ".

By subtracting (3.23) from (3.20), we find

$$
\begin{aligned}
\partial_{t}( & \left.\overline{S_{\lambda}(R)}-S_{\lambda}(\bar{R})\right)-\partial_{x}\left(c(u)\left(\overline{S_{\lambda}(R)}-S_{\lambda}(\bar{R})\right)\right) \\
= & \tilde{c}^{\prime}(u)\left\{-2 \overline{R S_{\lambda}(R)}+\overline{T_{\lambda}(R) R^{2}}+2 \bar{R} S_{\lambda}(\bar{R})-T_{\lambda}(\bar{R}) \bar{R}^{2}\right. \\
& \left.\quad+2 \bar{S}\left(\overline{S_{\lambda}(R)}-S_{\lambda}(\bar{R})\right)-\left(\overline{T_{\lambda}(R)}-T_{\lambda}(\bar{R})\right) \overline{S^{2}}+T_{\lambda}(\bar{R})\left(\overline{R^{2}}-\bar{R}^{2}\right)\right\} .
\end{aligned}
$$

But, by the explicit structures of $S_{\lambda}(\cdot)$ and $T_{\lambda}(\cdot)$, we find

$$
\begin{aligned}
& -2 \xi S_{\lambda}(\xi)+T_{\lambda}(\xi) \xi^{2}=-\lambda(\xi-\lambda)^{2} 1_{\xi \geqslant \lambda}-\lambda^{2}\left(\xi-T_{\lambda}(\xi)\right)+\lambda(\xi+\lambda)^{2} 1_{\xi \leqslant-\lambda}, \\
& 2 \bar{R} S_{\lambda}(\bar{R})-T_{\lambda}(\bar{R}) \bar{R}^{2}=\lambda(\bar{R}-\lambda)^{2} 1_{\bar{R} \geqslant \lambda}+\lambda^{2}\left(\bar{R}-T_{\lambda}(\bar{R})\right)-\lambda(\bar{R}+\lambda)^{2} 1_{\bar{R} \leqslant-\lambda} .
\end{aligned}
$$

Since

$$
\xi^{2}=2 S_{\lambda}(\xi)+(\xi-\lambda)^{2} 1_{\xi \geqslant \lambda}+(\xi+\lambda)^{2} 1_{\xi \leqslant-\lambda}
$$

we have

$$
\begin{aligned}
\overline{R^{2}}-(\bar{R})^{2}= & 2\left(\overline{S_{\lambda}(R)}-S_{\lambda}(\bar{R})\right)+\overline{(R-\lambda)^{2} 1_{R \geqslant \lambda}}-(\bar{R}-\lambda)^{2} 1_{\bar{R} \geqslant \lambda} \\
& +\overline{(R+\lambda)^{2} 1_{R \leqslant-\lambda}}-(\bar{R}+\lambda)^{2} 1_{\bar{R} \leqslant-\lambda .}
\end{aligned}
$$

Summing up (3.24)-(3.27), we find that

$$
\begin{aligned}
\partial_{t}\left(\overline{S_{\lambda}(R)}-S_{\lambda}(\bar{R})\right)-\partial_{x}\left(c(u)\left(\overline{S_{\lambda}(R)}-S_{\lambda}(\bar{R})\right)\right) \\
=\tilde{c}^{\prime}(u)\left\{\left(T_{\lambda}(\bar{R})-\lambda\right)\left[\overline{(R-\lambda)^{2} 1_{R} \geqslant \lambda}-(\bar{R}-\lambda)^{2} 1_{\bar{R} \geqslant \lambda}\right]\right. \\
\quad+\left(T_{\lambda}(\bar{R})+\lambda\right)\left[\overline{(R+\lambda)^{2} 1_{R \leqslant-\lambda}}-(\bar{R}+\lambda)^{2} 1_{\bar{R} \leqslant-\lambda}\right]-\lambda^{2}\left[T_{\lambda}(\bar{R})-\overline{T_{\lambda}(R)}\right] \\
\left.\quad-\left(\overline{T_{\lambda}(R)}-T_{\lambda}(\bar{R})\right) \overline{S^{2}}+2\left(\bar{S}+T_{\lambda}(\bar{R})\right)\left(\overline{S_{\lambda}(R)}-S_{\lambda}(\bar{R})\right)\right\} \\
=\tilde{c}^{\prime}(u)\left\{\left(T_{\lambda}(\bar{R})-\lambda\right) \overline{(R-\lambda)^{2} 1_{R} \geqslant \lambda}+\left(T_{\lambda}(\bar{R})+\lambda\right) \overline{(R+\lambda)^{2} 1_{R \leqslant-\lambda}}\right. \\
\left.\quad+\left(T_{\lambda}(\bar{R})-\overline{T_{\lambda}(R)}\right)\left(\overline{S^{2}}-\lambda^{2}\right)+2\left(\bar{S}+T_{\lambda}(\bar{R})\right)\left(\overline{S_{\lambda}(R)}-S_{\lambda}(\bar{R})\right)\right\} .
\end{aligned}
$$

We comment that the term $\left(T_{\lambda}(\bar{R})-\overline{T_{\lambda}(R)}\right) \overline{S^{2}}$ is difficult. We will use renormalization to handle it. The term

$$
G_{\lambda}:=\tilde{c}^{\prime}(u)\left\{\left(T_{\lambda}(\bar{R})-\lambda\right) \overline{(R-\lambda)^{2} 1_{R} \geqslant \lambda}+\left(T_{\lambda}(\bar{R})+\lambda\right) \overline{(R+\lambda)^{2} 1_{R \leqslant-\lambda}}-\left(T_{\lambda}(\bar{R})-\overline{T_{\lambda}(R)}\right) \lambda^{2}\right\}
$$

will be shown to be nonpositive. The remaining term of product in (3.28) is not hard. In step 5 we do some preparation for handling the two difficult terms. 
Step 5a. An inequality. We claim that

$$
\frac{1}{2}\left(\overline{T_{\lambda}(R)}-T_{\lambda}(\bar{R})\right)^{2} \leqslant \overline{S_{\lambda}(R)}-S_{\lambda}(\bar{R}) .
$$

In fact, by Cauchy inequality, we have

$$
{\overline{T_{\lambda}(R)}}^{2}=\left(\int T_{\lambda}(\xi) \mathrm{d} v_{t x}^{1}(\xi)\right)^{2} \leqslant \overline{\left(T_{\lambda}(R)\right)^{2}} .
$$

Using the identities

$$
\begin{aligned}
& \xi=T_{\lambda}(\xi)+(\xi-\lambda) 1_{\xi \geqslant \lambda}+(\xi+\lambda) 1_{\xi \leqslant-\lambda}, \\
& \bar{R}=T_{\lambda}(\bar{R})+(\bar{R}-\lambda) 1_{\bar{R} \geqslant \lambda}+(\bar{R}+\lambda) 1_{\bar{R} \leqslant-\lambda},
\end{aligned}
$$

we have

$$
\begin{aligned}
T_{\lambda}(\bar{R}) \overline{T_{\lambda}(R)}= & T_{\lambda}(\bar{R}) \bar{R}-T_{\lambda}(\bar{R}) \overline{(R-\lambda) 1_{R} \geqslant \lambda}-T_{\lambda}(\bar{R}) \overline{(R+\lambda) 1_{R \leqslant-\lambda}} \\
= & \left(T_{\lambda}(\bar{R})\right)^{2}-T_{\lambda}(\bar{R})\left(\overline{(R-\lambda) 1_{R} \geqslant \lambda}-(\bar{R}-\lambda) 1_{\bar{R} \geqslant \lambda}\right) \\
& -T_{\lambda}(\bar{R})\left(\overline{(R+\lambda) 1_{R \leqslant-\lambda}}-(\bar{R}+\lambda) 1_{\bar{R} \leqslant-\lambda}\right) .
\end{aligned}
$$

Thus

$$
\begin{aligned}
\left(\overline{T_{\lambda}(R)}-T_{\lambda}(\bar{R})\right)^{2}= & \overline{T_{\lambda}(R)^{2}}+\left(T_{\lambda}(\bar{R})\right)^{2}-2 T_{\lambda}(\bar{R}) \overline{T_{\lambda}(R)} \\
\leqslant & \overline{\left(T_{\lambda}(R)\right)^{2}}-\left(T_{\lambda}(\bar{R})\right)^{2}+2 T_{\lambda}(\bar{R})\left(\overline{(R-\lambda) 1_{R \geqslant \lambda}}-(\bar{R}-\lambda) 1_{\bar{R} \geqslant \lambda}\right) \\
& +2 T_{\lambda}(\bar{R})\left(\overline{(R+\lambda) 1_{R \leqslant-\lambda}}-(\bar{R}+\lambda) 1_{\bar{R} \leqslant-\lambda}\right) .
\end{aligned}
$$

Using

$$
S_{\lambda}(\xi)-\frac{1}{2}\left(T_{\lambda}(\xi)\right)^{2}=\lambda(\xi-\lambda) 1_{\xi \geqslant \lambda}-\lambda(\xi+\lambda) 1_{\xi \leqslant-\lambda},
$$

we then by (3.33) have

$$
\begin{aligned}
\overline{S_{\lambda}(R)}-S_{\lambda}(\bar{R})= & \frac{1}{2}\left(\overline{\left(T_{\lambda}(R)\right)^{2}}-\left(T_{\lambda}(\bar{R})\right)^{2}\right)+\lambda\left(\overline{(R-\lambda) 1_{R \geqslant \lambda}}-(\bar{R}-\lambda) 1_{\bar{R} \geqslant \lambda}\right) \\
& -\lambda\left(\overline{(R+\lambda) 1_{R \leqslant-\lambda}}-(\bar{R}+\lambda) 1_{\bar{R} \leqslant-\lambda}\right) \\
\geqslant & \frac{1}{2}\left(T_{\lambda}(\bar{R})-\overline{T_{\lambda}(R)}\right)^{2}+\left(\lambda-T_{\lambda}(\bar{R})\right)\left(\overline{(R-\lambda) 1_{R \geqslant \lambda}}-(\bar{R}-\lambda) 1_{\bar{R} \geqslant \lambda}\right) \\
& -\left(\lambda+T_{\lambda}(\bar{R})\right)\left(\overline{(R+\lambda) 1_{R \leqslant-\lambda}}-(\bar{R}+\lambda) 1_{\bar{R} \leqslant-\lambda}\right) \\
\geqslant & \frac{1}{2}\left(T_{\lambda}(\bar{R})-\overline{T_{\lambda}(R)}\right)^{2},
\end{aligned}
$$

where in the last step of the above we used the fact that $\left.(R-\lambda)\right|_{R \geqslant \lambda}$ is a convex function and $\left.(R+\lambda)\right|_{R \leqslant-\lambda}$ is a concave function. This proves (3.30).

Step 5b. A convergence. We shall need the almost everywhere convergence:

$$
\overline{T_{\lambda}(R)}-T_{\lambda}(\bar{R}) \rightarrow 0
$$

as $\lambda \rightarrow \infty$. We first have by Cauchy-Schwarz inequality that

$$
\left|\bar{R}-\overline{T_{\lambda}(R)}\right|=\int\left[(\xi-\lambda) 1_{\xi \geqslant \lambda}+(\xi+\lambda) 1_{\xi \leqslant-\lambda}\right] \mathrm{d} v_{t, x}^{1}(\xi) \leqslant \frac{1}{\lambda} \int \xi^{2} \mathrm{~d} v_{t, x}^{1}(\xi) .
$$


This together with (3.6) yield that

$$
\lim _{\lambda \rightarrow \infty}\left\|\bar{R}-\overline{T_{\lambda}(R)}\right\|_{L^{1}([0, T] \times \mathbb{R})}=0, \quad \forall T>0 .
$$

Similarly, we can prove that $\lim _{\lambda \rightarrow \infty}\left\|\bar{R}-T_{\lambda}(\bar{R})\right\|_{L^{1}([0, T] \times \mathbb{R})}=0$. Then by the triangle inequality, we obtain

$$
\lim _{\lambda \rightarrow \infty}\left\|\overline{T_{\lambda}(R)}-T_{\lambda}(\bar{R})\right\|_{L^{1}([0, T] \times \mathbb{R})}=0, \quad \forall T>0 .
$$

Step 5c. Another inequality. Let $\left(t_{0}, x_{0}\right) \in \mathbb{R}^{+} \times \mathbb{R}$ such that $c^{\prime}\left(u\left(t_{0}, x_{0}\right)\right)>0$, we claim that there is a neighborhood $\mathcal{N}\left(t_{0}, x_{0}\right)$ of $\left(t_{0}, x_{0}\right)$ and some positive constant $M\left(t_{0}, x_{0}\right)$ such that whenever $\lambda \geqslant M\left(t_{0}, x_{0}\right)$, the term $G_{\lambda}$ defined in (3.29) satisfies

$$
G_{\lambda} \leqslant 0, \quad(t, x) \in \mathcal{N}\left(t_{0}, x_{0}\right) .
$$

In fact, by Lemma 2.3, we find that there is a smaller neighborhood $\mathcal{N}\left(t_{0}, x_{0}\right)$ of $\left(t_{0}, x_{0}\right)$ and some positive constant $M\left(t_{0}, x_{0}\right)$ such that $R^{\varepsilon} \geqslant-M\left(t_{0}, x_{0}\right)$. Furthermore, for any $\phi(t, x) \in C_{c}^{\infty}\left(\mathcal{N}\left(t_{0}, x_{0}\right)\right), t_{0}>0$, there holds

$$
\begin{gathered}
\lim _{\varepsilon \rightarrow 0} \iint \phi(t, x) \tilde{c}^{\prime}\left(u^{\varepsilon}\right)\left(T_{\lambda}(\bar{R})+\lambda\right)\left(R^{\varepsilon}+\lambda\right)^{2} 1_{R^{\varepsilon} \leqslant-\lambda} \mathrm{d} x \mathrm{~d} t \\
\quad=\iint \phi(t, x) \tilde{c}^{\prime}(u)\left(T_{\lambda}(\bar{R})+\lambda\right)\left(\overline{R+\lambda)^{2} 1_{R \leqslant-\lambda}} \mathrm{d} x \mathrm{~d} t .\right.
\end{gathered}
$$

Thus by taking $\lambda>M\left(t_{0}, x_{0}\right)$ in the above, we find

$$
\tilde{c}^{\prime}(u)\left(T_{\lambda}(\bar{R})+\lambda\right)\left(\overline{R+\lambda)^{2} 1_{R \leqslant-\lambda}}=0, \quad \text { for }(t, x) \in \mathcal{N}\left(t_{0}, x_{0}\right) .\right.
$$

Similarly for $\lambda>M\left(t_{0}, x_{0}\right)$, there holds

$$
-\lambda^{2} \tilde{c}^{\prime}(u)\left(T_{\lambda}(\bar{R})-\overline{T_{\lambda}(R)}\right)=-\lambda^{2} \tilde{c}^{\prime}(u)\left(T_{\lambda}^{+}(\bar{R})-\overline{T_{\lambda}^{+}(R)}\right) \leqslant 0,
$$

for $(t, x) \in \mathcal{N}\left(t_{0}, x_{0}\right)$, where

$$
T_{\lambda}^{+}(\xi)= \begin{cases}\lambda, & \xi \geqslant \lambda \\ \xi, & \xi \leqslant \lambda\end{cases}
$$

which is a concave function. Trivially, there holds

$$
\tilde{c}^{\prime}(u)\left(T_{\lambda}(\bar{R})-\lambda\right)\left(\overline{R-\lambda)^{2} 1_{R} \geqslant \lambda} \leqslant 0, \quad \text { for }(t, x) \in \mathcal{N}\left(t_{0}, x_{0}\right) .\right.
$$

Summing up (3.36)-(3.39), we prove (3.35). Exactly similarly to the proof of (3.35), we can prove that (3.35) still holds when $c^{\prime}\left(u\left(t_{0}, x_{0}\right)\right)<0$.

Step 6a. Renormalization.

We let $f_{\lambda}(t, x):=\overline{S_{\lambda}(R)}-S_{\lambda}(\bar{R})$. Notice that $f_{\lambda}(t, x) \in L^{\infty}\left(R^{+}, L^{2}(\mathbb{R})\right)$ for any fixed $\lambda$. Thus by DiPernaLions folklore Lemma 2.3 (Lions [12]) and Lebesgue Dominated Convergence Theorem in the time direction again, we have

$$
\partial_{t} f_{\lambda}^{\varepsilon}-\partial_{x}\left(c(u) f_{\lambda}^{\varepsilon}\right)=G_{\lambda} * j_{\varepsilon}+\tilde{c}^{\prime}(u)\left\{2\left(\bar{S}+T_{\lambda}(\bar{R})\right) f_{\lambda}^{\varepsilon}+\overline{S^{2}}\left[T_{\lambda}(\bar{R})-\overline{T_{\lambda}(R)}\right]\right\}+\gamma_{\varepsilon},
$$

where $f_{\lambda}^{\varepsilon}(t, x):=\int_{\mathbb{R}} f_{\lambda}(t, y) j_{\varepsilon}(x-y) \mathrm{d} y$ and $\gamma_{\varepsilon} \rightarrow 0$ in $L_{\text {loc }}^{1}\left(\mathbb{R}^{+} \times \mathbb{R}\right)$. For any $\eta>0$, we multiply the above equation (3.40) with $\frac{1}{2}\left(f_{\lambda}^{\varepsilon}+\eta\right)^{-1 / 2}$ to yield

$$
\begin{aligned}
\partial_{t}\left(f_{\lambda}^{\varepsilon}+\eta\right)^{1 / 2}-\partial_{x}\left(c(u)\left(f_{\lambda}^{\varepsilon}+\eta\right)^{1 / 2}\right) \\
\left.=\left(\frac{1}{2} G_{\lambda} * j_{\varepsilon}+\tilde{c}^{\prime}(u)\left(\bar{R}+T_{\lambda}(\bar{R})\right) f_{\lambda}^{\varepsilon}\right)\left(f_{\lambda}^{\varepsilon}+\eta\right)^{-1 / 2}-2 \tilde{c}^{\prime}(u)(\bar{R}-\bar{S})\right)\left(f_{\lambda}^{\varepsilon}+\eta\right)^{1 / 2} \\
\quad+\frac{1}{2} \tilde{c}^{\prime}(u) \overline{S^{2}}\left(f_{\lambda}^{\varepsilon}+\eta\right)^{-1 / 2}\left(T_{\lambda}(\bar{R})-\overline{T_{\lambda}(R)}\right)+\frac{1}{2}\left(f_{\lambda}^{\varepsilon}+\eta\right)^{-1 / 2} \gamma_{\varepsilon} .
\end{aligned}
$$


By taking $\varepsilon \rightarrow 0$ in (3.41), we find

$$
\begin{aligned}
\partial_{t}( & \left.f_{\lambda}+\eta\right)^{1 / 2}-\partial_{x}\left(c(u)\left(f_{\lambda}+\eta\right)^{1 / 2}\right) \\
= & \left.\left(\frac{1}{2} G_{\lambda}+\tilde{c}^{\prime}(u)\left(\bar{R}+T_{\lambda}(\bar{R})\right) f_{\lambda}\right)\left(f_{\lambda}+\eta\right)^{-1 / 2}-2 \tilde{c}^{\prime}(u)(\bar{R}-\bar{S})\right)\left(f_{\lambda}+\eta\right)^{1 / 2} \\
& +\frac{1}{2} \tilde{c}^{\prime}(u) \overline{S^{2}}\left(f_{\lambda}+\eta\right)^{-1 / 2}\left(T_{\lambda}(\bar{R})-\overline{T_{\lambda}(R)}\right) .
\end{aligned}
$$

Moreover, by (3.30), we find that

$$
\overline{S^{2}}\left(f_{\lambda}+\eta\right)^{-1 / 2}\left|T_{\lambda}(\bar{R})-\overline{T_{\lambda}(R)}\right| \leqslant 2 \overline{S^{2}} .
$$

Thus, by (3.34) and Lebesgue Dominated Convergence Theorem, we find for any $T>0$ that

$$
\lim _{\lambda \rightarrow \infty}\left\|\overline{S^{2}}\left(f_{\lambda}+\eta\right)^{-1 / 2}\left(T_{\lambda}(\bar{R})-\overline{T_{\lambda}(R)}\right)\right\|_{L^{1}([0, T] \times \mathbb{R})}=0 .
$$

Trivially, by (3.6) and Lebesgue Dominated Convergence Theorem, we have

$$
\lim _{\lambda \rightarrow \infty} f_{\lambda}(t, x)=\frac{1}{2}\left(\overline{R^{2}}-\bar{R}^{2}\right)=: f(t, x) .
$$

Moreover, by summing up (3.42) with (3.43), we find that: $\left\{G_{\lambda}\left(f_{\lambda}+\eta\right)^{-1 / 2}\right\}$ is uniformly bounded in $H_{\text {loc }}^{-1}((0, T) \times$ $\mathbb{R})+L^{\infty}\left([0, T], L^{1}(\mathbb{R})\right)$ with respect to $\lambda$ and $\eta$ for all $T>0$. Thus by a diagonal process for $T$, we can find two sequences, $\left\{\lambda_{i}\right\},\left\{\eta_{j}\right\}$, with $\lambda_{i} \rightarrow \infty, \eta_{j} \rightarrow 0$ as $i, j \rightarrow \infty$, such that for all $T>0$, there holds

$$
G_{\lambda_{i}}\left(f_{\lambda_{i}}+\eta_{j}\right)^{-1 / 2} \rightarrow G
$$

in the sense of distributions as $i \rightarrow \infty, j \rightarrow \infty$. Thus by summing up (3.42)-(3.46), taking $\lambda=\lambda_{i}, \eta=\eta_{j}$ in (3.42), and let $i \rightarrow \infty$, then $j \rightarrow \infty$, we find

$$
\partial_{t} g-\partial_{x}(c(u) g)=\frac{1}{2} G+2 \tilde{c}^{\prime}(u) \bar{S} g
$$

where $g(t, x):=\sqrt{f(t, x)}$.

Step 6b. Nonpositivity of $G$. Now let us assume that $c^{\prime}(\cdot) \geqslant 0$ and $c^{\prime}\left(u_{0}(\cdot)\right)>0$. By Lemma 2.4 and a similar proof of (3.35), for any small enough $t_{0}$ and any $(\bar{t}, \bar{x}) \in \mathbb{R}^{+} \times \mathbb{R}$ with $\bar{t}>t_{0}$, there is a neighborhood $\mathcal{N}(\bar{t}, \bar{x})$ such that (3.35) holds for $\lambda>\bar{M}$. Thus for all test function $0 \leqslant \varphi \in C_{c}^{\infty}(\mathcal{N}(\bar{t}, \bar{x}))$, we have

$$
\iint_{\mathcal{N}(\bar{t}, \bar{x})} \varphi G \mathrm{~d} x \mathrm{~d} t=\lim _{j \rightarrow \infty} \lim _{i \rightarrow \infty} \iint_{\mathcal{N}(\bar{t}, \bar{x})} \varphi G_{\lambda_{i}}\left(f_{\lambda_{i}}+\eta_{j}\right)^{-1 / 2} \mathrm{~d} x \mathrm{~d} t \leqslant 0,
$$

which implies that $\left.G\right|_{\mathcal{N}(\bar{t}, \bar{x})} \leqslant 0$ in the sense of distributions. Thus, by the local property of the distributions, we find that

$$
\left.G\right|_{\left(t_{0}, \infty\right) \times \mathbb{R}} \text { is a distribution, and }\left.G\right|_{\left(t_{0}, \infty\right) \times \mathbb{R}} \leqslant 0 .
$$

Then $\left.G\right|_{\left(t_{0}, \infty\right) \times \mathbb{R}}$ is actually a nonpositive Radon measure.

By summing up (3.47), (3.49), we find

$$
\partial_{t} g-\partial_{x}(c(u) g) \leqslant 2 \tilde{c}^{\prime}(u) \bar{S} g, \quad\left(t_{0}, \infty\right) \times \mathbb{R} .
$$


Step 6c. Re-renormalization. Notice that $g(t, x) \in L^{\infty}\left(\mathbb{R}^{+}, L^{2}(\mathbb{R})\right)$. Thus again by DiPerna-Lions folklore Lemma 2.3 (Lions [12]), we obtain

$$
\partial_{t} g^{\varepsilon}-\partial_{x}\left(c(u) g^{\varepsilon}\right) \leqslant 2 \tilde{c}^{\prime}(u) \bar{S} g^{\varepsilon}+\gamma_{\varepsilon}, \quad \text { in }\left(t_{0}, \infty\right) \times \mathbb{R}
$$

where $0 \leqslant g^{\varepsilon}(t, x):=\int g(t, y) j_{\varepsilon}(x-y) \mathrm{d} y$ and $\gamma_{\varepsilon}(t, x) \rightarrow 0$ in $L_{\text {loc }}^{1}\left(\mathbb{R}^{+} \times \mathbb{R}\right)$.

On the other hand, parallel to the proof of (3.18), we can prove that

$$
\partial_{t} \bar{S}+\partial_{x}(c(u) \bar{S})=-\tilde{c}^{\prime}(u)\left(\overline{R-S)^{2}} .\right.
$$

Moreover, by the third equation of (2.2), there holds

$$
2 c(u) u_{x}=\bar{R}-\bar{S} \text {. }
$$

Subtracting (3.52) from (3.18), we obtain

$$
\partial_{t}(\bar{R}-\bar{S})-\partial_{x}(c(u)(\bar{R}+\bar{S}))=0 .
$$

Substituting (3.53) into the above equation, we find

$$
\partial_{x}\left(c(u)\left(2 u_{t}-(\bar{R}+\bar{S})\right)\right)=0 ;
$$

that is

$$
u_{t}=\frac{1}{2}(\bar{R}+\bar{S})
$$

Dividing (3.51) with $\sqrt{c(u)}$, we obtain

$$
\partial_{t}\left(\frac{g^{\varepsilon}}{\sqrt{c(u)}}\right)-\partial_{x}\left(\sqrt{c(u)} g^{\varepsilon}\right) \leqslant \frac{\gamma_{\varepsilon}}{\sqrt{c(u)}}, \quad \text { in }\left(t_{0}, \infty\right) \times \mathbb{R} .
$$

Taking $\varepsilon \rightarrow 0$ in (3.55), we obtain

$$
\partial_{t}\left(\frac{g}{\sqrt{c(u)}}\right)-\partial_{x}(\sqrt{c(u)} g) \leqslant 0, \quad \text { in }\left(t_{0}, \infty\right) \times \mathbb{R} .
$$

Step 6d. The precompactness.

Firstly by the definition of $g(t, x)$, we have $g(t, x) \in L^{\infty}\left(\mathbb{R}^{+}, L^{2}(\mathbb{R})\right)$. So, if we take $\phi(x) \in C_{c}^{\infty}(\mathbb{R})$ with $\phi(x)=1$ for $|x| \leqslant 1$ and $\phi(x)=0$ for $|x|>2$, then $c^{-1 / 2} g \phi\left(\frac{x}{n}\right) \in L^{\infty}\left(\mathbb{R}^{+}, L^{1}(\mathbb{R})\right)$. We note then that almost all $t \in \mathbb{R}$ is a Lebesgue point of $\int_{\mathbb{R}}\left(c^{-1 / 2}(u) g\right)(t, x) \phi\left(\frac{x}{n}\right) \mathrm{d} x$.

By the energy inequality (3.1) and the proof of (6.39) in [18], which imply that

$$
\lim _{t \rightarrow 0} \int_{\mathbb{R}}\left(\int_{\mathbb{R}}(\xi-\bar{R})^{2} \mathrm{~d} \nu_{t x}^{1}(\xi)+\int_{\mathbb{R}}(\eta-\bar{S})^{2} \mathrm{~d} v_{t x}^{2}(\eta)\right) \mathrm{d} x=0,
$$

we find that

$$
\lim _{t \rightarrow 0} \int_{\mathbb{R}} \frac{g}{\sqrt{c(u)}}(t, x) \phi\left(\frac{x}{n}\right) \mathrm{d} x=0 .
$$

Furthermore, motivated by [10] and [13], let us take $\bar{t}$ to be one of its Lebesgue point of $\int_{\mathbb{R}}\left(c^{-1 / 2}(u) g\right)(t, x) \times$ $\phi\left(\frac{x}{n}\right) \mathrm{d} x$, and take $\psi^{\delta}(t) \in C_{c}^{\infty}\left(\mathbb{R}^{+}\right)$such that

$$
\begin{aligned}
& \psi^{\delta}(t)= \begin{cases}0, & t \leqslant \frac{\delta}{2} \text { or } t \geqslant \bar{t}+\delta, \\
1, & \delta \leqslant t \leqslant \bar{t}-\delta,\end{cases} \\
& 0 \leqslant \partial_{t} \psi^{\epsilon}(t) \leqslant \frac{C}{\delta}, \quad t \in[0, \delta], \quad-\partial_{t} \psi^{\epsilon}(t) \geqslant \frac{C}{\delta}, \quad t \in[\bar{t}-\delta, \bar{t}+\delta] .
\end{aligned}
$$


Let us multiplying $\psi^{\delta}(t) \phi\left(\frac{x}{n}\right)$ to (3.56) and integrating over $\left(\frac{\delta}{4}, \infty\right) \times \mathbb{R}$ to yield

$$
\begin{aligned}
& \frac{C}{\delta} \int_{\bar{t}-\delta}^{\bar{t}+\delta} \int_{\mathbb{R}} \frac{g}{\sqrt{c(u)}} \phi\left(\frac{x}{n}\right) \mathrm{d} x \mathrm{~d} t \leqslant-\int_{\bar{t}-\delta}^{\bar{t}+\delta} \int_{\mathbb{R}} \partial_{t} \psi^{\delta} \frac{g}{\sqrt{c(u)}} \phi\left(\frac{x}{n}\right) \mathrm{d} x \mathrm{~d} t \\
& \leqslant \int_{\delta / 4}^{\delta} \int_{\mathbb{R}} \partial_{t} \psi^{\delta} \frac{g}{\sqrt{c(u)}} \phi\left(\frac{x}{n}\right) \mathrm{d} x \mathrm{~d} t+\frac{1}{n} \int_{0}^{\bar{t}+\delta} \int_{\mathbb{R}} \psi^{\delta}\left|\phi^{\prime}\left(\frac{x}{n}\right)\right| \sqrt{c(u)} g \mathrm{~d} x \mathrm{~d} t \\
& \leqslant C / \delta \int_{\delta / 4}^{\delta} \int_{\mathbb{R}} \frac{g}{\sqrt{c(u)}} \phi\left(\frac{x}{n}\right) \mathrm{d} x+\frac{C}{n} \int_{0}^{\bar{t}+\delta} \int_{\mathbb{R}}\left|\phi^{\prime}\left(\frac{x}{n}\right)\right| g \mathrm{~d} x \mathrm{~d} t .
\end{aligned}
$$

Taking $\delta \rightarrow 0$ in (3.59) and using (3.58), we find

$$
C \int_{\mathbb{R}} \frac{g}{\sqrt{c(u)}}(\bar{t}, x) \phi\left(\frac{x}{n}\right) \mathrm{d} x \leqslant \frac{C}{n} \int_{0}^{\bar{t}} \int_{\mathbb{R}}\left|\phi^{\prime}\left(\frac{x}{n}\right)\right| g \mathrm{~d} x \mathrm{~d} t \leqslant \frac{C}{\sqrt{n}},
$$

which together Fatou's Lemma yields that

$$
\int_{\mathbb{R}} \frac{g}{\sqrt{c(u)}}(\bar{t}, x) \mathrm{d} x=0 .
$$

Due to arbitrariness of $\bar{t}$, we obtain

$$
g(t, x)=0, \quad \text { a.e. }(t, x) \in \mathbb{R}^{+} \times \mathbb{R} .
$$

Hence $f(t, x)=0$ a.e. $(t, x) \in \mathbb{R}^{+} \times \mathbb{R}$ and therefore $v_{t x}^{1}(\xi)=\delta_{\bar{R}(t, x)}(\xi)$. Similarly, we can prove that $v_{t x}^{2}(\eta)=$ $\delta_{\bar{S}(t, x)}(\eta)$. This completes the proof of the lemma.

Remark 3.1. By (3.35) and (3.48), the assumptions that $c^{\prime}(\cdot) \geqslant 0$ and $c^{\prime}\left(u_{0}\right)>0$ were used only to prove that the distribution $G \leqslant 0$ around the set $\left\{(t, x): c^{\prime}(u(t, x))=0\right\}$. That is to say, if we can prove this without the restriction, we can actually improve Theorem 1.1 for general wave speed $c(u)$ with $c^{\prime}(\cdot)$ changing sign.

Now, we prove Theorem 1.1.

Proof of Theorem 1.1. Firstly, by (2.37), there holds (1.8), and by (3.53) and (3.54), we find

$$
\bar{R}=\partial_{t} u+c(u) \partial_{x} u, \quad \bar{S}=\partial_{t} u-c(u) \partial_{x} u .
$$

Secondly, by (3.18), (3.52), and Lemma 3.2, we find that

$$
\left\{\begin{array}{l}
\partial_{t} \bar{R}-\partial_{x}(c(u) \bar{R})=-\tilde{c}^{\prime}(u)(\bar{R}-\bar{S})^{2}, \\
\partial_{t} \bar{S}+\partial_{x}(c(u) \bar{S})=-\tilde{c}^{\prime}(u)(\bar{R}-\bar{S})^{2}
\end{array}\right.
$$

hold in the sense of distributions. Summing up the two equations of (3.63) and using (3.62), we find that there holds (1.7). Moreover, by (3.1) and (3.62), there holds (1.6). By (2.19) and (3.53), there holds (1.10). This completes the proof of Theorem 1.1. 


\section{Acknowledgements}

Ping Zhang is supported by NSF of China under Grant 10131050 and 10276036, the innovation grants from Chinese Academy of Sciences. Yuxi Zheng is supported in part by NSF DMS-0305497 and 0305114. This work was done when Ping Zhang visited Penn State University. He would like to thank the department for its hospitality. Both authors would also like to thank Professors Andy Majda, Craig Evans, Fanghua Lin, Bob Glassey, and John Hunter for their constant interest in our work.

\section{References}

[1] R.J. DiPerna, P.L. Lions, Ordinary differential equations, transport theory and Sobolev spaces, Invent. Math. 98 (1989) $511-547$.

[2] R.J. DiPerna, A. Majda, Oscillations and concentrations in weak solutions of the incompressible fluid equations, Comm. Math. Phys. 108 (1987) 667-689.

[3] L.C. Evans, Weak Convergence Methods for Nonlinear Partial Differential Equations, CBMS Regional Conf. Ser. in Math., vol. 74, Amer. Math. Soc., Providence, RI, 1990.

[4] P. Gerard, Microlocal defect measures, Comm. Partial Differential Equations 16 (1991) 1761-1794.

[5] R.T. Glassey, J.K. Hunter, Y. Zheng, Singularities in a nonlinear variational wave equation, J. Differential Equations 129 (1996) 49-78.

[6] R.T. Glassey, J.K. Hunter, Y. Zheng, Singularities and oscillations in a nonlinear variational wave equation, in: J. Rauch, M. Taylor (Eds.), IMA, vol. 91, Springer, 1997.

[7] A. Grundland, E. Infeld, A family of nonlinear Klein-Gordon equations and their solutions, J. Math. Phys. 33 (1992) $2498-2503$.

[8] J.K. Hunter, R.A. Saxton, Dynamics of director fields, SIAM J. Appl. Math. 51 (1991) 1498-1521.

[9] J.K. Hunter, Y. Zheng, On a nonlinear hyperbolic variational equation I and II, Arch. Rational Mech. Anal. 129 (1995) $305-353$ and 355-383.

[10] S. Jiang, P. Zhang, On the 3-D axi-symmetric solutions to the compressible Navier-Stokes equations, J. Math. Pures Appl. (9) 82 (2003) 949-973.

[11] J.L. Joly, G. Métivier, J. Rauch, Focusing at a point and absorption of nonlinear oscillations, Trans. Amer. Math. Soc. 347 (1995) $3921-$ 3970.

[12] P.L. Lions, Mathematical Topics in Fluid Mechanics, vol. 1, Incompressible Models, Lecture Series in Mathematics and its Applications, vol. 3, Clarendon Press, Oxford, 1996.

[13] P.L. Lions, Mathematical Topics in Fluid Mechanics, vol. 2, Compressible Models, Lecture Series in Mathematics and its Applications, vol. 6, Clarendon Press, Oxford, 1998.

[14] R.A. Saxton, Dynamic instability of the liquid crystal director, in: W.B. Lindquist (Ed.), Current Progress in Hyperbolic Systems, in: Contemp. Math., vol. 100, Amer. Math. Soc., Providence, RI, 1989, pp. 325-330.

[15] L. Tartar, Compensated compactness and applications to partial differential equations, in: R.J. Knops (Ed.), Nonlinear Analysis and Mechanics: Heriot-Watt Symposium, in: Res. Notes Math., vol. 39, Pitman, 1979.

[16] L. Tartar, H-measures, a new approach for studying homogenisation oscillations and concentration effects in partial differential equations, Proc. Roy. Soc. Edinburg Sect. A 115 (1990) 193-230.

[17] L.C. Young, Lectures on the Calculus of Variations and Optimal Control Theory, Saunders, Philadelphia, 1969.

[18] P. Zhang, Y. Zheng, Rarefactive solutions to a nonlinear variational wave equation, Comm. Partial Differential Equations 26 (2001) $381-$ 420.

[19] P. Zhang, Y. Zheng, Existence and uniqueness of solutions to an asymptotic equation of a variational wave equation with general data, Arch. Rational Mech. Anal. 155 (2000) 49-83.

[20] P. Zhang, Y. Zheng, Singular and rarefactive solutions to a nonlinear variational wave equation, Chinese Ann. Math. Ser. B 22B (2000) $159-170$.

[21] P. Zhang, Y. Zheng, Weak solutions to a nonlinear variational wave equation, Arch. Rational Mech. Anal. 166 (2003) $303-319$.

[22] H. Zorski, E. Infeld, New soliton equations for dipole chains, Phys. Rev. Lett. 68 (1992) 1180-1183. 